\title{
THE ZERO NORM SUBSPACE OF BOUNDED COHOMOLOGY OF ACYLINDRICALLY HYPERBOLIC GROUPS
}

\author{
F. FRANCESCHINI, R. FRIGERIO, M. B. POZZETTI, AND A. SISTO
}

\begin{abstract}
We construct combinatorial volume forms of hyperbolic three manifolds fibering over the circle. These forms define non-trivial classes in bounded cohomology. After introducing a new seminorm on exact bounded cohomology, we use these combinatorial classes to show that, in degree 3, the zero norm subspace of the bounded cohomology of an acylindrically hyperbolic group is infinite dimensional. In the appendix we use the same techniques to give a cohomological proof of a lower bound, originally due to Brock, on the volume of the mapping torus of a cobounded pseudo-Anosov homeomorphism of a closed surface in terms of its Teichmüller translation distance.
\end{abstract}

Even for very well-studied groups such as non-abelian free groups, the task of computing the bounded cohomology in higher degrees is still challenging. In degree 2, the technology of quasimorphisms has been extensively exploited to construct non-trivial bounded cohomology classes (see e.g. Bro81, EF97, Fuj98, BF02, Fuj00 for the case of trivial coefficients, and [HO13, BBF16, CFI16] for more general coefficient modules). On the other hand, in higher degrees both constructing bounded cocycles and showing that such cocycles define non-trivial bounded cohomology classes is definitely non-trivial. For example, as far as the authors know, in the case of non-abelian free groups, non-trivial bounded classes in degree 3 have been constructed only with the help of hyperbolic geometry (see e.g. [Som97a, Som97b, Som97c]), and it is still a major open question whether the fourth bounded cohomology of non-abelian free groups vanishes or not.

The purpose of this paper is twofold. First, we construct a discrete 3dimensional volume form on a class of free-by-cyclic groups. Then, building on results from [FPS15], we exploit our construction to show that, for every acylindrically hyperbolic group, the space of bounded classes with vanishing seminorm is infinite dimensional in degree 3.

Following a suggestion by Mladen Bestvina, our construction is based on a suitable relative version of Mineyev's bicombing on hyperbolic groups [Min01, which is due to Groves and Manning [GM08] and Franceschini [Fra]. Dealing with a discrete volume form rather than with differential forms allows us to provide a somewhat unified version of the arguments developed in [Som97c], where some essential estimates make use of a careful comparison between

Date: July 21, 2018. 
the volume forms arising from the hyperbolic and the singular Sol structure supported by hyperbolic 3-manifolds that fiber over the circle. We hope that our combinatorial arguments, although clearly inspired by their differential counterpart, could be more easily extended to wider classes of groups and, maybe, even to higher degrees.

Bounded cohomology of discrete groups. Let $\Gamma$ be a group. We briefly recall the definition of bounded cohomology of $\Gamma$ (with trivial real coefficients), referring the reader to Section 1 for more details. We denote by $C^{n}(\Gamma)$ the set of real-valued homogeneous $n$-cochains on $\Gamma$, and for every $\varphi \in C^{n}(\Gamma)$ we set

$$
\|\varphi\|_{\infty}=\sup \left\{\left|\varphi\left(g_{0}, \ldots, g_{n}\right)\right| \mid\left(g_{0}, \ldots, g_{n}\right) \in \Gamma^{n+1}\right\} \in[0, \infty] .
$$

We denote by $C_{b}^{n}(\Gamma) \subseteq C^{n}(\Gamma)$ the subspace of bounded cochains, and by $C^{n}(\Gamma)^{\Gamma}, C_{b}^{n}(\Gamma)^{\Gamma}$ the subspaces of invariant (bounded) cochains. The cohomology of the complex $C_{b}^{*}(\Gamma)^{\Gamma}$ is the bounded cohomology $H_{b}^{*}(\Gamma)$ of $\Gamma$. The norm $\|\cdot\|_{\infty}$ on $C_{b}^{n}(\Gamma)$ induces a seminorm on $H_{b}^{n}(\Gamma)$ that is usually called the Gromov seminorm.

The inclusion of (invariant) bounded cochains into ordinary cochains induces the comparison map $c^{n}: H_{b}^{n}(\Gamma) \rightarrow H^{n}(\Gamma)$. The kernel of $c^{n}$ is the set of bounded cohomology classes whose representatives are exact, and it is denoted by $E H_{b}^{n}(\Gamma)$. By definition, a class $\alpha \in E H_{b}^{n+1}(\Gamma)$ is represented by a bounded cocycle $z=\delta \varphi \in C_{b}^{n+1}(\Gamma)^{\Gamma}$, where $\varphi \in C^{n}(\Gamma)^{\Gamma}$ is a (possibly unbounded) cochain. In other words, if we define the space $\mathrm{QZ}^{n}(\Gamma) \subseteq C^{n}(\Gamma)$ of $n$-quasi-cocycles as the subset of cochains having bounded differential, then the differential induces a surjection $\mathrm{QZ}^{n}(\Gamma)^{\Gamma} \longrightarrow E H_{b}^{n+1}(\Gamma)$.

We denote by $N^{n}(\Gamma)$ the subspace of $H_{b}^{n}(\Gamma)$ given by elements with vanishing Gromov seminorm. It is easy to show that $N^{n}(\Gamma) \subseteq E H_{b}^{n}(\Gamma)$ for every $n \in \mathbb{N}$ (see Lemma 1.2). It was proved by Matsumoto and Morita MM85] and independently by Ivanov [Iva90 that $N^{2}(\Gamma)=0$ for every group $\Gamma$. On the other hand, Soma proved that $N^{3}\left(F_{2}\right) \neq 0$ [Som98], and that the dimension of $N^{3}\left(\Gamma_{g}\right)$ has the cardinality of the continuum [Som97c], where $F_{2}$ and $\Gamma_{g}$ denote respectively the free group on two generators and the fundamental group of a closed orientable surface of genus $g \geq 2$.

Main results. In this paper we extend Soma's results as follows:

Theorem 1. Suppose that $\Gamma$ is acylindrically hyperbolic. Then the dimension of $N^{3}(\Gamma)$ has the cardinality of the continuum.

A group $\Gamma$ is acylindrically hyperbolic if it admits a non-elementary acylindrical action on a Gromov hyperbolic space Osi13. The class of acylindrically hyperbolic groups includes many examples of interest: non-elementary hyperbolic and relatively hyperbolic groups DGO16, the mapping class group of all but finitely many surfaces of finite type [DGO16, Theorem 2.19], $\operatorname{Out}\left(F_{n}\right)$ for $n \geq 2$ [DGO16, Theorem 2.20], groups acting geometrically on a proper $\mathrm{CAT}(0)$ space with a rank one isometry (Sis11] and DGO16, 
Theorem 2.22]), fundamental groups of several graphs of groups [MO13, small cancellation groups GS14, finitely presented residually finite groups with positive first $\ell^{2}$-Betti number as well as groups of deficiency at least 2 Osi15, and many more. In particular, Theorem 1 widely generalizes Soma's previously mentioned results.

In order to prove Theorem 1 we proceed as follows. We introduce a new seminorm $\|\cdot\|_{\infty, 0}$ on exact bounded cohomology, which satisfies the inequality $\|\cdot\|_{\infty, 0} \geq\|\cdot\|_{\infty}$ : for every finite subset $S$ of $\Gamma$ and class $\alpha \in$ $E H_{b}^{n}(\Gamma)$ we set

$$
\|\alpha\|_{S}=\inf \left\{\|\delta \varphi\|_{\infty}\left|\varphi \in C^{n-1}(\Gamma)^{\Gamma},[\delta \varphi]=\alpha, \varphi\right|_{S^{n}}=0\right\},
$$

and we define

$$
\|\alpha\|_{\infty, 0}=\sup \left\{\|\alpha\|_{S}, S \subseteq \Gamma, S \text { finite }\right\} \in[0,+\infty] .
$$

We denote by $N_{0}^{n}(\Gamma)$ the subspace of elements $\alpha \in E H_{b}^{n}(\Gamma)$ such that $\|\alpha\|_{\infty, 0}=0$, so that $N_{0}^{n}(\Gamma) \subseteq N^{n}(\Gamma)$ for every $n \in \mathbb{N}$. The key step in our proof of Theorem 1 is then provided by the following:

Theorem 2. The dimension of $N_{0}^{3}\left(F_{2}\right)$ has the cardinality of the continuum.

This already implies Theorem 1 for non-abelian free groups (and, therefore, for all groups that admit an epimorphism on $F_{2}$, e.g. for surface groups). We then exploit results from FPS15 to reduce the general case to the case of free groups. In fact, an acylindrically hyperbolic group $\Gamma$ contains a hyperbolically embedded subgroup $H$ which is virtually freenon-abelian DGO16, Osi13 (in fact, random subgroups satisfy this property [MS17]). Moreover, [FPS15, Corollary 1.2] implies that the inclusion $H \hookrightarrow \Gamma$ induces a surjection of $E H_{b}^{3}(\Gamma)$ onto $E H_{b}^{3}(H)$, which we know to be infinite-dimensional from Theorem 2, This does not quite suffice to conclude, since we do not know whether the surjection $E H_{b}^{3}(\Gamma) \rightarrow E H_{b}^{3}(H)$ does restrict to a surjection $N^{3}(\Gamma) \rightarrow N^{3}(H)$. This last fact would be true provided that the map $E H_{b}^{3}(\Gamma) \rightarrow E H_{b}^{3}(H)$ is undistorted, according to the following:

Definition 3. A map $f: V \rightarrow W$ between seminormed vector spaces is undistorted if there exists $k \geq 0$ such that for every $\alpha \in f(V)$ there exists $\beta \in V$ with $f(\beta)=\alpha$ and $\|\beta\| \leq k \cdot\|\alpha\|$.

Unfortunately, we are not able to show that the surjection $E H_{b}^{3}(\Gamma) \rightarrow$ $E H_{b}^{3}(H)$ is undistorted with respect to Gromov seminorms. In fact, Remark 2.8 says that this cannot be achieved at the level of quasi-cocycles, and therefore undistortion is a rather delicate matter related to coboundaries, which makes it far from clear that this should even be true. Nevertheless, in Section 2 we prove the following:

Theorem 4. Let $H$ be hyperbolically embedded in $\Gamma$, let $n \geq 2$ and suppose that $H^{n-1}(H)$ is finite-dimensional. If we endow both $E H_{b}^{n}(\Gamma)$ and $E H_{b}^{n}(H)$ 
with the seminorm $\|\cdot\|_{\infty, 0}$, then the inclusion $H \hookrightarrow \Gamma$ induces an undistorted surjection

$$
E H_{b}^{n}(\Gamma) \longrightarrow E H_{b}^{n}(H)
$$

This immediately implies that $\operatorname{dim} N^{3}(\Gamma) \geq \operatorname{dim} N_{0}^{3}(\Gamma) \geq \operatorname{dim} N_{0}^{3}(H)$, thus allowing us to deduce Theorem 1 from Theorem 2. Indeed, much more is true: due to the definition of $\|\cdot\|_{\infty, 0}$, the fact that $\operatorname{dim} N_{0}^{3}(\Gamma)$ is infinitedimensional implies that there are many non-trivial classes in $E H_{b}^{3}(\Gamma)$ with vanishing seminorm, each of which can be represented by cocycles that vanish on arbitrarily big subsets of $\Gamma$. This quite counterintuitive phenomenon vividly illustrates the failing of excision for bounded cohomology.

Quasi-cocycles. Bounded cohomology is often computed via suitable resolutions, that allow to better exploit the geometry of the group under consideration. For example, suppose that $\Gamma$ acts on a set $X$. Then we denote by $C^{n}(\Gamma \curvearrowright X)$ (resp. $\left.C_{b}^{n}(\Gamma \curvearrowright X)\right)$ the space of maps (resp. bounded maps) from $X^{n+1}$ to $\mathbb{R}$, endowed with the $\Gamma$-action defined by

$$
g \cdot \varphi\left(x_{0}, \ldots, x_{n}\right)=\varphi\left(g^{-1} x_{0}, \ldots, g^{-1} x_{n}\right) .
$$

The obvious differential $\delta: C^{n}(\Gamma \curvearrowright X) \rightarrow C^{n+1}(\Gamma \curvearrowright X)$ preserves both $\Gamma$-invariance and boundedness of cochains, so one can define the bounded cohomology $H_{b}^{*}(\Gamma \curvearrowright X)$ as the cohomology of the complex $C_{b}^{*}(\Gamma \curvearrowright X)^{\Gamma}$ of invariant bounded cochains.

The $\ell^{\infty}$-norm $\|\cdot\|_{\infty}$ on $C_{b}^{n}(\Gamma \curvearrowright X)$ induces an $\ell^{\infty}$-seminorm on $H_{b}^{n}(\Gamma \curvearrowright$ $X)$, which is still denoted by $\|\cdot\|_{\infty}$. Moreover, if the action of $\Gamma$ on $X$ is free, then $H_{b}^{n}(\Gamma \curvearrowright X)$ is canonically isometrically isomorphic to $H_{b}^{n}(\Gamma)$ for every $n \in \mathbb{N}$ (see Lemma 1.1). In particular, $N^{3}(\Gamma)$ is canonically isomorphic to the subspace of elements of $H_{b}^{3}(\Gamma \curvearrowright X)$ with vanishing seminorms. Every element with vanishing seminorm is exact (see Lemma 1.2), so it can be represented by a quasi-cocycle. We are thus lead to investigate the space

$$
\mathrm{QZ}^{n}(\Gamma \curvearrowright X)=\left\{\varphi \in C^{n}(\Gamma \curvearrowright X) \mid\|\delta \varphi\|_{\infty}<\infty\right\}
$$

of quasi-cocycles defined on $X$ : namely, in order to prove that $H_{b}^{3}(\Gamma)$ contains many elements with vanishing seminorm, we will construct an uncountable family of invariant 2-quasi-cocycles whose differential defines linearly independent bounded cohomology classes.

A crucial notion that keeps track of the seminorm of classes induced by quasi-cocycles is the defect: just as in the case of quasi-morphisms, the defect of a quasi-cocycle $\varphi \in \mathrm{QZ}^{n}(\Gamma \curvearrowright X)$ is given by

$$
\operatorname{def}(\varphi)=\|\delta \varphi\|_{\infty} .
$$

A combinatorial volume form on hyperbolic 3-manifolds fibering over the circle. Let us now look more closely at the case we are interested in. Let $\Gamma_{0}=F_{2}$ be the free group generated by the elements $a, b$, and let us identify $\Gamma_{0}$ with the fundamental group of the punctured torus $\Sigma$, in such a way that the conjugacy class of the commutator $[a, b]=a^{-1} b^{-1} a b$ 
corresponds to the isotopy class of a simple closed curve winding around the puncture. We fix a group automorphism $\psi: \Gamma_{0} \rightarrow \Gamma_{0}$ induced by a pseudo-Anosov homeomorphism $f: \Sigma \rightarrow \Sigma$. The automorphism $\psi$ preserves the conjugacy class of the commutator $[a, b]$, so, up to conjugacy, we may suppose that $\psi([a, b])=[a, b]$. The mapping torus

$$
M=\Sigma \times[0,1] / \sim, \quad(x, 0) \sim(f(x), 1)
$$

has fundamental group isomorphic to the semidirect product $\Gamma=\Gamma_{0} \rtimes_{\psi} \mathbb{Z}$, where the generator $t$ of $\mathbb{Z}$ acts on $\Gamma_{0}$ as follows: $\operatorname{tgt}^{-1}=\psi(g)$ for every $g \in \Gamma_{0}$. The (cusp) subgroup $H$ of $\Gamma$ is the subgroup generated by $t$ and (the image of) $[a, b] \in \Gamma_{0}$, and it is isomorphic to $\mathbb{Z} \oplus \mathbb{Z}$.

Recall that the pair $(\Gamma, H)$ is relatively hyperbolic, either by Thurston's hyperbolization for manifolds fibering over the circle Ota96 and a fundamental result by Farb [Far98], or just by a combination theorem for relative hyperbolicity [MR08, Theorem 4.9].

Starting from a Cayley graph of $\Gamma$, one can construct a cusped graph $X$ by gluing a copy of a combinatorial horoball based on $H$ to each left coset of $H$ in $\Gamma$; we outline the construction in Section 3. It was first described by Groves and Manning in GM08], and a similar construction is described in Bow12.

The group $\Gamma$ acts freely on $X$ by isometries, therefore the bounded cohomology of $\Gamma_{0}$ can be isometrically computed by the complex $C_{b}^{*}\left(\Gamma_{0} \curvearrowright X\right)^{\Gamma_{0}}$. Moreover, being obtained by adding horoballs to (the Cayley graph of) a relatively hyperbolic group, the graph $X$ is Gromov hyperbolic, and supports a quasi-geodesic homological bicombing with useful filling properties (see Section 3). Indeed, $X$ is quasi-isometric to the hyperbolic 3-space, and the bicombing may be exploited to construct a combinatorial version of the hyperbolic volume form. In fact, since the cochains arising in our argument must all be $\Gamma_{0}$-invariant, the combinatorial cocycles we construct should be thought of as volume forms on the differential counterpart of $X / \Gamma_{0}$, that is the infinite cyclic covering $M_{0}$ of $M$ associated to $\Gamma_{0}<\Gamma=\pi_{1}(M)$.

As it is customary when dealing with "quasifications" of algebraic or differential notions, the direct construction of a volume cocycle on $X$ runs into difficulties, due to the fact that the coarse version of a cocycle needs not be a cocycle. Therefore, in Section 4 we rather construct a $\Gamma_{0}$-invariant primitive of a volume form. Such primitive is a quasi-cocycle, and its differential (which is automatically closed) provides a combinatorial version of the volume form on $M_{0} \cong \Sigma \times \mathbb{R}$. Following Soma's strategy, in order to construct an infinite-dimensional subspace of $E H^{3}\left(\Gamma_{0}\right)$ out of this primitive, we just consider the suitably chosen collection of quasi-cocycles obtained by taking the product of the original primitive with a collection of real functions on $M_{0} \cong \Sigma \times \mathbb{R}$. These functions are themselves constructed by composing the projection $\Sigma \times \mathbb{R} \rightarrow \mathbb{R}$ with Lipschitz maps of $\mathbb{R}$ into itself. The outcome of this procedure is summarized by the following result, which provides the key ingredient for the proof of Theorem 2 ; 
Theorem 5. Let $\mathcal{L}(\mathbb{Z}, \mathbb{R})$ be the space of Lipschitz real functions on $\mathbb{Z}$. There exist a constant $C>0$ and a linear map

$$
\alpha: \mathcal{L}(\mathbb{Z}, \mathbb{R}) \rightarrow \mathrm{QZ}_{\text {alt }}^{2}\left(\Gamma_{0} \curvearrowright X\right)^{\Gamma_{0}}
$$

such that the following conditions hold:

(1) $\| \delta \alpha(f)) \|_{\infty} \leq C \cdot \operatorname{Lip}(f)$ for every $f \in \mathcal{L}(\mathbb{Z}, \mathbb{R})$;

(2) $[\delta \alpha(f)]=0$ in $H_{b}^{3}\left(\Gamma_{0} \curvearrowright X\right) \cong H_{b}^{3}\left(\Gamma_{0}\right)$ if and only if $f$ is bounded.

Volumes of mapping tori. We believe that the techniques developed in this paper, and especially the combinatorial description of a volume form, will have application in other contexts as well. As a first example in this direction, in the appendix we give a cohomological proof of a volume estimate for hyperbolic 3-manifolds fibering over the circle, under a coboundedness assumption. Recall that a pseudo-Anosov homeomorphism $\psi: \Sigma_{g} \rightarrow \Sigma_{g}$ is $\epsilon$-cobounded if, denoting by $l$ its axis in the Teichmüller space endowed with the Teichmüller metric, the projection of $l$ is contained in the $\epsilon$-thick part $\mathcal{M}_{g}^{\epsilon}$ of the moduli space. We denote by $\tau(\psi)$ the translation length of $\psi$ on the Teichmüller space endowed with the Teichmüller metric.

Theorem 6. There exists a constant $C>0$ depending only on $\epsilon$ and $g$ such that, for any $\epsilon$-cobounded pseudo-Anosov $\psi: \Sigma_{g} \rightarrow \Sigma_{g}$, we have

$$
\operatorname{vol}\left(M_{\psi}\right) \geq C \tau(\psi) .
$$

This result was originally proven by Brock with completely different techniques Bro03b. In fact, we emphasize that our proof actually gives an estimate on the simplicial volume of $M_{\psi}$, and we then deduce the volume estimate from the well-known proportionality between volume and simplicial volume for hyperbolic manifolds. However, in no other part of the proof we use the fact that $M_{\psi}$ is hyperbolic.

We decided to include such a result only in an appendix because the setting is slightly different from the rest of the paper. Since we only deal with compact manifolds, many of the technicalities involved in the main paper are not needed for this application. For this reason, a reader interested only in the construction of a combinatorial cocycle representing the volume form might want to read the appendix first.

Tl;dr: the definition of the quasi-cocycles. For future reference and to help the reader find the relevant definitions, we list here all notions involved in the construction of our quasi-cocycles, and we give the definition of the quasi-cocycles themselves.

- $\Gamma_{0}$ is the free group on two generators, $a, b$.

- $\psi: \Gamma_{0} \rightarrow \Gamma_{0}$ is an automorphism induced by a pseudo-Anosov, and it preserves the commutator $[a, b]$.

- $\Gamma$ is the semidirect product $\Gamma_{0} \rtimes_{\psi} \mathbb{Z}$, and $H<\Gamma$ is the subgroup generated by $[a, b]$ and the stable letter $t$.

- $X$ is the cusped graph of $(\Gamma, H)$ (Definition 3.1) , which is $\delta$-hyperbolic. Vertices of $X$ are pairs $(g, n)$ with $g \in G, n \in \mathbb{N}$. 
- $p: X^{(0)} \rightarrow \Gamma_{0}$ is defined by $\psi\left(g_{0} t^{k}, n\right)=g_{0}$, where $g_{0} \in \Gamma_{0}$. Also, $\theta: X^{(0)} \rightarrow \mathbb{Z}$ is defined by $\theta\left(g_{0} t^{k}, n\right)=k$, where $g_{0} \in \Gamma_{0}$.

- $\rho: \Gamma_{0} \rightarrow \operatorname{Isom}^{+}\left(\mathbb{H}^{2}\right)$ is a hyperbolization, and $[a, b]$ fixes $\bar{q} \in \partial \mathbb{H}^{2}$. For $x_{0}, x_{1}, x_{2}$ vertices of $X$, the sign $\epsilon\left(x_{0}, x_{1}, x_{2}\right)$ is $1,-1$ or 0 depending on the orientation of the ideal triangle of $\mathbb{H}^{2}$ with vertices $\rho\left(p\left(x_{i}\right)\right) \bar{q}$ (Subsection 4.2).

- $\mathcal{X}$ is the Rips complex on $X$ with constant $\kappa \geq 4 \delta+6$ (Definition 3.6).

- $\varphi$ is a relative filling map, i.e. a map from $X^{3}$ to 2-cochains of $\mathcal{X}$ (Proposition 3.11).

Let now $f: \mathbb{Z} \rightarrow \mathbb{R}$ be Lipschitz.

- The simplicial 2-cochain $F_{f}$ on $\mathcal{X}$ (Definition 4.3) is the one such that, if $\sigma$ is a 2 -simplex in $\mathcal{X}$ with vertices $\left(x_{0}, x_{1}, x_{2}\right) \in X^{3}$, then

$$
F_{f}(\sigma)=\varepsilon\left(x_{0}, x_{1}, x_{2}\right) \frac{\sum_{i=0}^{2} f\left(\theta\left(x_{i}\right)\right)}{3} .
$$

- Finally, the quasi-cocycle $\alpha_{f}: X^{3} \rightarrow \mathbb{R}$ is defined by

$$
\alpha_{f}\left(x_{0}, x_{1}, x_{2}\right)=F_{f}\left(\varphi\left(x_{0}, x_{1}, x_{2}\right)\right) .
$$

Proposition 4.3 says that $\alpha_{f}$ is indeed a quasi-cocycle, and that its defect is bounded by a universal constant times the Lipschitz constant of $f$. Proposition 4.6 says that the coboundary $\delta \alpha_{f}$ is trivial in bounded cohomology if and only if $f$ is bounded.

Open questions and directions for further research. Is quasification indeed essential in order to prove Theorem 2] Surprisingly enough, it seems that studying genuine differential forms on hyperbolic manifolds is much harder than working with quasi-cocycles on discrete models for $F_{2}$. For example, if $M_{0} \cong \Sigma \times \mathbb{R}$ is the hyperbolic manifold introduced above, where $\Sigma$ is a punctured torus, integration over straight simplices induces a map from the space of pointwise bounded differential 3 -forms on $M_{0}$ to bounded group cochains of degree 3 . Understanding the kernel of this map is unexpectedly difficult, and this implies that it is not trivial to detect when distinct differential forms represent the same bounded class, i.e. how much freedom one can enjoy in varying the differential representatives of a fixed bounded class. We refer the reader to BI07, Wie12] for a discussion of this topic. In KK15 Kim and Kim proved, for example, that if $M$ is a complete, connected, oriented, locally symmetric space of infinite volume, then the Cheeger isoperimetric constant of $M$ is positive if and only if the Riemannian volume form on $M$ admits a bounded primitive. They also showed that if $M$ is a complete, connected, oriented, $\mathbb{R}$-rank one locally symmetric space of infinite volume with dimension at least 3 , then the volume form of $M$ defines a non-trivial bounded cohomology class if and only if the Cheeger constant of $M$ vanishes. We pose here the following: 
Question 7. Let $n \geq 3$ and let $M$ be a hyperbolic $n$-manifold of infinite volume with vanishing Cheeger constant. Is it possible to characterize the space of $n$-forms on $M$ admitting a bounded primitive? For example, is it true that a compactly supported $n$-form on $M$ admits a bounded primitive?

This question is tacitly faced in [Som97c] in the case when $M$ is the cyclic covering of a 3-manifold fibering over the circle with fiber a closed surface. Soma's analysis involves a careful study of the relationship between the hyperbolic and the singular Sol volume forms supported by such a manifold. Adapting his arguments to the case when the fiber is a punctured surface seems very delicate.

Monod and Shalom showed the importance of bounded cohomology with coefficients in $\ell^{2}(\Gamma)$ in the study of rigidity of $\Gamma$ NM04, MS06], and proposed the condition $H_{b}^{2}\left(\Gamma, \ell^{2}(\Gamma)\right) \neq 0$ as a cohomological definition of negative curvature for groups. More in general, bounded cohomology with coefficients in $\ell^{p}(\Gamma), 1 \leq p<\infty$ has been widely studied as a powerful tool to prove (super)rigidity results (see e.g. Ham08, CFI16). It is still unknown whether $H_{b}^{3}\left(F_{2}, \ell^{2}\left(F_{2}\right)\right)$ vanishes or not. We hope that our combinatorial approach to the construction of non-trivial classes (with trivial real coefficients) could be of use in the context of more general coefficient modules.

Plan of the paper. In Section 1 we recall some basic facts on bounded cohomology, and introduce the various (co)homological complexes we will need in the paper. In Section 2 we introduce the seminorm $\|\cdot\|_{\infty, 0}$ and prove Theorem 4 building on results from [FPS15]. We also show how Theorem 1 may be reduced to Theorem 2, Following GM08 and [Fra], in Section 3 we describe a combinatorial bicombing with good filling properties on a suitably chosen Rips complex associated to a relatively hyperbolic pair. In Section 4 we construct a family of 3-dimensional combinatorial volume forms on the free group on two generators, and we prove Theorems 5 and 2 . Finally, in the appendix, we discuss applications of our techniques to obtain bounds on the volume of compact hyperbolic manifolds and prove Theorem 6 .

Acknowledgements. We thank Mladen Bestvina for an important suggestion that made it possible to construct a combinatorial version of Soma's classes, and Ken Bromberg for very useful discussions. Part of this work was carried out during the conference "Ventotene 2015 - Manifolds and Groups". Beatrice Pozzetti was partially supported by the SNF grant P2EZP2_159117. This material is based upon work supported by the National Science Foundation under Grant No. DMS-1440140 while the fourth author was in residence at the Mathematical Sciences Research Institute in Berkeley, California, during the Fall 2016 semester. The third and fourth author would like to thank the Isaac Newton Institute for Mathematical Sciences, Cambridge, for support and hospitality during the programme "Non-Positive Curvature Group Actions and Cohomology" where work on this paper was undertaken. This work was supported by EPSRC grant no EP/K032208/1. 


\section{Preliminaries on BOUnded COHOMOlOGY}

Let $\Gamma$ be a group and let $X$ be a set on which $\Gamma$ acts on the left. We set

$$
C^{n}(\Gamma \curvearrowright X)=\left\{\varphi: X^{n+1} \rightarrow \mathbb{R}\right\}
$$

and we endow $C^{n}(\Gamma \curvearrowright X)$ with the left $\Gamma$-action defined by

$$
g \cdot \varphi\left(x_{0}, \ldots, x_{n}\right)=\varphi\left(g^{-1} x_{0}, \ldots, g^{-1} x_{n}\right) .
$$

For every $n \in \mathbb{N}$ we also define the differential $\delta: C^{n}(\Gamma \curvearrowright X) \rightarrow C^{n+1}(\Gamma \curvearrowright$ $X$ ) by setting

$$
\delta \varphi\left(x_{0}, \ldots, x_{n+1}\right)=\sum_{i=0}^{n+1}(-1)^{i} \varphi\left(x_{0}, \ldots, \widehat{x}_{i}, \ldots, x_{n+1}\right),
$$

and we put on $C^{n}(\Gamma \curvearrowright X)$ the norm defined by

$$
\|\varphi\|_{\infty}=\sup \left\{\left|\varphi\left(x_{0}, \ldots, x_{n}\right)\right|,\left(x_{0}, \ldots, x_{n}\right) \in X^{n+1}\right\} \in[0,+\infty] .
$$

We denote by $C_{b}^{n}(\Gamma \curvearrowright X) \subseteq C^{n}(\Gamma \curvearrowright X)$ the subspace of bounded cochains, and we observe that $\|\cdot\|_{\infty}$ restricts to a finite norm on $C_{b}^{n}(\Gamma \curvearrowright X)$.

If $V$ is a vector space endowed with a linear $\Gamma$-action, we denote by $V^{\Gamma} \subset V$ the subspace of elements that are fixed by every element of $\Gamma$. The differential defined above commutes with the action of $\Gamma$ and sends bounded cochains to bounded cochains. Therefore, we can consider the cohomology of the complexes $C^{n}(\Gamma \curvearrowright X)^{\Gamma}$ and $C_{b}^{n}(\Gamma \curvearrowright X)^{\Gamma}$, which we denote respectively by $H^{n}(\Gamma \curvearrowright X)$ and $H_{b}^{n}(\Gamma \curvearrowright X)$. If $X=\Gamma$, endowed with the left action by translations, one gets back the usual (bounded) cohomology $H_{(b)}^{*}(\Gamma)$ of $\Gamma$.

For every basepoint $x \in X$, we consider the $\Gamma$-equivariant chain map

$$
\begin{gathered}
w_{x}^{*}: C_{b}^{*}(\Gamma \curvearrowright X) \rightarrow C_{b}^{*}(\Gamma), \\
w_{x}^{n}(\varphi)\left(g_{0}, \ldots, g_{n}\right)=\varphi\left(g_{0} x, \ldots, g_{n} x\right) .
\end{gathered}
$$

With a slight abuse, we denote by $w_{x}^{n}$ also the induced map $w_{x}^{n}: H_{b}^{n}(\Gamma \curvearrowright$ $X) \rightarrow H_{b}^{n}(\Gamma)$ on bounded cohomology.

Lemma 1.1. Suppose that the action of $\Gamma$ on $X$ is free. Then for every $x \in$ $X$ the map $w_{x}^{n}: H_{b}^{n}(\Gamma \curvearrowright X) \rightarrow H_{b}^{n}(\Gamma)$ is a natural isometric isomorphism.

Proof. Free actions are very special instances of amenable actions, so the conclusion follows e.g. from [Mon01, Theorem 7.5.3].

1.1. The predual chain complex. In order to show that the cocycles we are going to construct are non-trivial, we will need to evaluate them on appropriate chains. Let us fix an action of a group $\Gamma$ on a set $X$ as in the previous section. For every $n \geq 0$ we denote by $C_{n}(X)$ the real vector space with basis $X^{n+1}$. Elements of $X^{n+1}$ will be often called $n$-simplices, since they are the $n$-simplices of the full simplicial complex with vertices in $X$. As usual, we say that an $n$-simplex is supported on a subset $S \subseteq X$ if all its vertices lie in $S$, and the subspace of $C_{n}(X)$ generated by simplices 
supported on $S$ is denoted by $C_{n}(S)$. We also endow $C_{n}(X)$ with the $\ell^{1}$ norm defined by

$$
\left\|\sum_{\bar{x} \in X^{n+1}} a_{\bar{x}} \bar{x}\right\|_{1}=\sum_{\bar{x} \in X^{n+1}}\left|a_{\bar{x}}\right| .
$$

If $\bar{x}=\left(x_{0}, \ldots, x_{n}\right) \in C_{n}(X)$, we denote by $\partial_{j} \bar{x}=\left(x_{0}, \ldots, \widehat{x_{j}}, \ldots, x_{n}\right) \in$ $C_{n-1}(X)$ the $j$-th face of $\bar{x}$, and we set $\partial \bar{x}=\sum_{j=0}^{n}(-1)^{j} \partial_{j} \bar{x}$. Observe that it readily follows from the definitions that the diagonal $\Gamma$-action on $X^{n+1}$ induces an isometric $\Gamma$-action on $C_{n}(X)$. We denote by $C_{n}(\Gamma \curvearrowright X)$ the normed space $C_{n}(X)$ equipped with this action.

The dual notion to cochain invariance is chain coinvariance. We define the space of coinvariants of $C_{n}(\Gamma \curvearrowright X)$ as the quotient space

$$
C_{n}(\Gamma \curvearrowright X)_{\Gamma}=C_{n}(\Gamma \curvearrowright X) / W
$$

where $W$ is the subspace of $C_{n}(\Gamma \curvearrowright X)$ spanned by the elements of the form $g \cdot c-c$, as $c$ varies in $C_{n}(\Gamma \curvearrowright X)$ and $g$ varies in $\Gamma$. We endow $C_{n}(\Gamma \curvearrowright X)_{\Gamma}$ with the quotient seminorm (which is a norm). Since the $\Gamma$ action on $C_{n}(\Gamma \curvearrowright X)$ commutes with the boundary operator, $C_{*}(\Gamma \curvearrowright X)_{\Gamma}$ is naturally a chain complex, whose homology will be denoted by

$$
H_{*}(\Gamma \curvearrowright X) .
$$

The $\ell^{1}$-norm on $C_{n}(\Gamma \curvearrowright X)_{\Gamma}$ induces a seminorm on $H_{*}(\Gamma \curvearrowright X)$, which will still be denoted by $\|\cdot\|_{1}$.

Since invariant cochains vanish on the subspace $W$ previously defined, evaluation of cochains on chains induces a pairing

$$
\langle\cdot, \cdot\rangle: C^{n}(\Gamma \curvearrowright X)^{\Gamma} \times C_{n}(\Gamma \curvearrowright X)_{\Gamma} \rightarrow \mathbb{R},
$$

which in turn induces pairings

$$
\begin{aligned}
& \langle\cdot, \cdot\rangle: H^{n}(\Gamma \curvearrowright X) \times H_{n}(\Gamma \curvearrowright X) \rightarrow \mathbb{R}, \\
& \langle\cdot, \cdot\rangle: H_{b}^{n}(\Gamma \curvearrowright X) \times H_{n}(\Gamma \curvearrowright X) \rightarrow \mathbb{R} .
\end{aligned}
$$

It readily follows from the definitions that

$$
\langle\alpha, \beta\rangle \leq\|\alpha\|_{\infty} \cdot\|\beta\|_{1}
$$

for every $\alpha \in H_{b}^{n}(\Gamma \curvearrowright X), \beta \in H_{n}(\Gamma \curvearrowright X)$. As a first application of the pairing between homology and cohomology, we show that bounded coclasses with vanishing seminorm are exact:

Lemma 1.2. We have $N^{n}(\Gamma) \subseteq E H_{b}^{n}(\Gamma)$.

Proof. By the Universal Coefficient Theorem, the pairing $\langle\cdot, \cdot\rangle: H^{n}(\Gamma) \times$ $H_{n}(\Gamma) \rightarrow \mathbb{R}$ induces an isomorphism between $H^{n}(\Gamma)$ and the dual of $H_{n}(\Gamma)$. Therefore, in order to conclude it is sufficient to observe that, if $c^{n}: H_{b}^{n}(\Gamma) \rightarrow$ $H^{n}(\Gamma)$ is the comparison map, then

$$
\left|\left\langle c^{n}(\alpha), \beta\right\rangle\right|=|\langle\alpha, \beta\rangle| \leq\|\alpha\|_{\infty}\|\beta\|_{1}=0
$$

for every $\alpha \in N^{n}(\Gamma), \beta \in H_{n}(\Gamma)$. 
1.2. Degenerate chains and alternating cochains. In later computations it will be convenient to neglect degenerate simplices (i.e. simplices with non-pairwise distinct vertices). To this aim, let us denote by $\mathfrak{S}_{n+1}$ the group of permutations of the set $\{0, \ldots, n\}$, and by $\operatorname{sgn}(\sigma)= \pm 1$ the sign of $\sigma$, for every $\sigma \in \mathfrak{S}_{n+1}$.

Then we may define an alternating linear operator alt ${ }_{n}: C_{n}(\Gamma \curvearrowright X) \rightarrow$ $C_{n}(\Gamma \curvearrowright X)$ by setting, for every $\bar{x}=\left(x_{0}, \ldots, x_{n}\right) \in X^{n+1}$,

$$
\operatorname{alt}_{n}(\bar{x})=\frac{1}{(n+1) !} \sum_{\sigma \in \mathfrak{S}_{n+1}} \operatorname{sgn}(\sigma)\left(x_{\sigma(0)}, \ldots, x_{\sigma(n)}\right) .
$$

We say that a chain $c \in C_{n}(\Gamma \curvearrowright X)$ is degenerate if $\operatorname{alt}_{n}(c)=0$, and we denote by $D_{n}(\Gamma \curvearrowright X)$ the subspace of degenerate chains. We observe that $D_{n}(\Gamma \curvearrowright X)$ contains (strictly, unless $X$ is a point) the space spanned by degenerate simplices.

It is immediate to check that alt ${ }_{*}$ commutes with the boundary operator and with the action of $\Gamma$. Therefore, it descends to a chain map alt $_{*}: C_{*}(\Gamma \curvearrowright X)_{\Gamma} \rightarrow C_{*}(\Gamma \curvearrowright X)_{\Gamma}$, which will still be denoted by alt ${ }_{*}$. If $D_{n}(\Gamma \curvearrowright X)_{\Gamma}$ denotes the image of $D_{n}(\Gamma \curvearrowright X)$ in $C_{n}(\Gamma \curvearrowright X)_{\Gamma}$, we then define reduced chains by setting

$$
\begin{aligned}
C_{*}(\Gamma \curvearrowright X)_{\mathrm{red}} & =C_{*}(\Gamma \curvearrowright X) / D_{*}(\Gamma \curvearrowright X), \\
C_{*}(\Gamma \curvearrowright X)_{\mathrm{red}, \Gamma} & =C_{*}(\Gamma \curvearrowright X)_{\Gamma} / D_{*}(\Gamma \curvearrowright X)_{\Gamma} .
\end{aligned}
$$

It is well known that the homology of the complex $C_{*}(\Gamma \curvearrowright X)_{\text {red, } \Gamma \text {, en- }}$ dowed with the obvious quotient seminorm, is isometrically isomorphic to $H_{n}(\Gamma \curvearrowright X)$ : indeed, this easily follows from the fact that alternation is homotopic to the identity (on any complex where it is defined), and norm non-increasing (see e.g. [FM11, Appendix B]).

Dually, one may define alternating cochains by setting, for every $\varphi \in$ $C^{n}(\Gamma \curvearrowright X)$,

$$
\operatorname{alt}^{n}(\varphi)(\bar{x})=\varphi\left(\operatorname{alt}_{n}(\bar{x})\right)
$$

for every $\bar{x} \in X^{n+1}$. The map alt ${ }^{n}$ commutes with the differential and with the action of $\Gamma$, thus defining a norm non-increasing chain self-map of the complex $C^{*}(\Gamma \curvearrowright X)$. We denote by

$$
C_{\text {alt }}^{*}(\Gamma \curvearrowright X)=\operatorname{alt}^{*}\left(C^{*}(\Gamma \curvearrowright X)\right)
$$

the space of alternating cochains, and we set

$$
C_{b, \text { alt }}^{*}(\Gamma \curvearrowright X)=C_{\text {alt }}^{*}(\Gamma \curvearrowright X) \cap C_{b}^{*}(\Gamma \curvearrowright X) .
$$

Again, the inclusion of alternating cochains into generic cochains induces an isometric isomorphism between the cohomology of the complex $C_{b, \text { alt }}^{*}(\Gamma \curvearrowright X)$ and $H_{b}^{*}(\Gamma \curvearrowright X)$. Moreover, since alternating cochains vanish on degenerate chains, there is a well-defined pairing

$$
\langle\cdot, \cdot\rangle: C_{b, \text { alt }}^{n}(\Gamma \curvearrowright X)^{\Gamma} \times C_{n}(\Gamma \curvearrowright X)_{\mathrm{red}, \Gamma} \rightarrow \mathbb{R},
$$


which, under the identifications previously mentioned, induces the pairing between $H_{b}^{n}(\Gamma \curvearrowright X)$ and $H_{n}(\Gamma \curvearrowright X)$ introduced above.

We will denote by $\mathrm{QZ}_{\mathrm{alt}}^{n}(\Gamma \curvearrowright X)$ the space of alternating quasi-cocycles on $X$, i.e. the set of alternating cochains with bounded differential.

1.3. Simplicial (co)chains. In this paper we will study the cochain modules $C^{*}(\Gamma \curvearrowright X), C_{b}^{*}(\Gamma \curvearrowright X)$ in the case when $X$ is the set of vertices of a suitably augmented Cayley graph of $\Gamma$ (see Section 3). A key step in our arguments will be based on the fact that $\Gamma$ is relatively hyperbolic, which implies that $\Gamma$ satisfies (relative) isoperimetric inequalities in every degree. In order to deal with higher dimensional fillings, it will be convenient, rather than considering cochains in $C^{n}(\Gamma \curvearrowright X)$ (or $\left.C_{b}^{n}(\Gamma \curvearrowright X)\right)$, to consider simplicial cochains on suitably defined simplicial complexes related to $X$ (like the augmented Cayley graph having $X$ as set of vertices, or some Rips complex over $X$ ).

For every simplicial complex $Y$, we denote by $\left(C_{*}^{\Delta}(Y), \partial\right)$ the chain complex of real simplicial chains on $Y$, endowed with the $\ell^{1}$-norm $\|\cdot\|_{1}$ such that

$$
\left\|\sum_{i \in I} a_{i} \sigma_{i}\right\|_{1}=\sum_{i \in I}\left|a_{i}\right|
$$

for every reduced sum $\sum_{i \in I} a_{i} \sigma_{i} \in C_{*}^{\Delta}(Y)$. The module $C_{n}^{\Delta}(Y)$ is the real vector space with basis

$$
\left\{\left(y_{0}, \ldots, y_{n}\right) \mid\left\{y_{0}, \ldots, y_{n}\right\} \text { is a simplex of } Y\right\}
$$

(in particular, every $n$-simplex of $Y$ gives rise to $(n+1)$ ! simplices in $C_{n}^{\Delta}(Y)$, and to many other degenerate ones in degree bigger than $n)$. As it is customary in the literature, we denote by $\left[y_{0}, \ldots, y_{n}\right]$ (rather than by $\left(y_{0}, \ldots, y_{n}\right)$ ) the elements of the canonical basis of $C_{n}^{\Delta}(Y)$. If $\left[y_{0}, \ldots, y_{n}\right]$ is any such element, then we set $\operatorname{Supp}\left(\left[y_{0}, \ldots, y_{n}\right]\right)=\left\{y_{0}, \ldots, y_{n}\right\} \subseteq Y^{(0)}$, and if $c=$ $\sum_{i \in I} a_{i} \sigma_{i}$ is a chain in reduced form, then we set $\operatorname{Supp}(c)=\bigcup_{i \in I} \operatorname{Supp}\left(\sigma_{i}\right)$. Just as above, we define a chain map $\operatorname{alt}_{n}: C_{n}^{\Delta}(Y) \rightarrow C_{n}^{\Delta}(Y)$ by setting

$$
\operatorname{alt}_{n}\left(\left[y_{0}, \ldots, y_{n}\right]\right)=\frac{1}{(n+1) !} \sum_{\sigma \in \mathfrak{S}_{n+1}} \operatorname{sgn}(\sigma)\left(\left[y_{\sigma(0)}, \ldots, y_{\sigma(n)}\right]\right)
$$

for every $\left[y_{0}, \ldots, y_{n}\right] \in C_{n}^{\Delta}(Y)$.

A chain $c \in C_{n}(Y)$ is degenerate if $\operatorname{alt}_{n}(c)=0$, and one may define the complex $C_{*}^{\Delta}(Y)_{\text {red }}$ of reduced simplicial chains as the quotient of $C_{*}(Y)$ by the subspace of degenerate chains. We will simply denote by $\left[y_{0}, \ldots, y_{n}\right]$ (and call it a "simplex") also the class of $\left[y_{0}, \ldots, y_{n}\right]$ in $C_{n}^{\Delta}(Y)_{\text {red }}$, so that, for example, we will be allowed to write that $\left[y_{0}, y_{1}\right]=-\left[y_{1}, y_{0}\right]$ in $C_{1}^{\Delta}(Y)_{\text {red }}$. If one fixes a total ordering $<$ on the set of vertices of $Y$, then a basis of $C_{n}^{\Delta}(Y)_{\text {red }}$ is given by the classes of the non-degenerate elements $\left[y_{0}, \ldots, y_{n}\right] \in$ $C_{n}^{\Delta}(Y)$ such that $y_{0}<\ldots<y_{n}$. We say that a simplex $\left\{y_{0}, \ldots, y_{n}\right\}$ appears in a reduced chain $\bar{c} \in C_{n}^{\Delta}(Y)_{\text {red }}$ if, when writing $\bar{c}$ as a linear combination of 
the elements of the above basis, the coefficient of the unique element corresponding to $\left\{y_{0}, \ldots, y_{n}\right\}$ is not null. We then define the $\operatorname{support} \operatorname{Supp}(\bar{c})$ of $\bar{c}$ as the union of the sets of vertices of all the simplices appearing in $\bar{c}$. Equivalently, $\operatorname{Supp}(\bar{c})$ is the smallest possible support of any chain $c \in C_{n}^{\Delta}(Y)$ projecting to $\bar{c}$.

Also observe that the $\ell_{1}$-norm on $C_{n}^{\Delta}(Y)$ induces an $\ell^{1}$-norm on $C_{n}^{\Delta}(Y)_{\text {red }}$, that will still be denoted by $\|\cdot\|_{1}$.

If $\Gamma$ acts on $Y$ via simplicial automorphisms, then we denote by $C_{*}^{\Delta}(Y)_{\Gamma}$ the complex of coinvariants of $C_{*}^{\Delta}(Y)$. Just as before, $C_{*}^{\Delta}(Y)_{\Gamma}$ is the quotient of $C_{*}^{\Delta}(Y)$ by the submodule generated by the chains of the form $(c-g \cdot c), c \in C_{*}^{\Delta}(Y), g \in \Gamma$. The chain map alt ${ }_{*}$ commutes with the action of $\Gamma$, thus descending to a map alt ${ }_{*}: C_{*}^{\Delta}(Y)_{\Gamma} \rightarrow C_{*}^{\Delta}(Y)_{\Gamma}$, which will

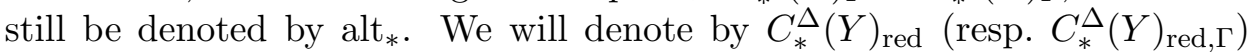
the complex of reduced (resp. reduced and coinvariant) cochains, i.e. the quotient of $C_{*}^{\Delta}(Y)$ (resp. of $C_{*}^{\Delta}(Y)_{\Gamma}$ ) by the kernel of the alternation map.

It is well known that, if $Y$ is contractible and $\Gamma$ acts freely on $Y$, the homology of the complexes $C_{*}^{\Delta}(Y)_{\Gamma}, C_{*}^{\Delta}(Y)_{\text {red, } \Gamma}$ is (not isometrically!) isomorphic to the homology of $\Gamma$. One may wonder whether also the computation of bounded cohomology could take place in the context of simplicial cochains. However, this is almost never the case: for example, if $Y / \Gamma$ is compact, then every invariant simplicial cochain on $Y$ is bounded, while there may well exist cohomology classes in $H^{n}(\Gamma)$ which do not admit any bounded representative.

\section{Controlled extensions of QuAsi-COCyCles}

This section is devoted to the description of some elementary properties of the norm $\|\cdot\|_{\infty, 0}$ defined in the introduction, and to the proof of Theorem 4. We fix a group $\Gamma$, and we work with the standard resolution $\left(C_{b}^{n}(\Gamma), \delta\right)$ computing $H_{b}^{n}(\Gamma)$.

2.1. The seminorm $\|\cdot\|_{\infty, 0}$. Recall from the introduction that, for every class $\alpha \in E H_{b}^{n+1}(\Gamma)$, we have set

$$
\|\alpha\|_{\infty, 0}=\sup \left\{\|\alpha\|_{S}, S \subseteq \Gamma, S \text { finite }\right\} \in[0,+\infty],
$$

where

$$
\|\alpha\|_{S}=\inf \left\{\|\delta \varphi\|_{\infty}\left|\varphi \in C^{n}(\Gamma)^{\Gamma},[\delta \varphi]=\alpha, \varphi\right|_{S}=0\right\} .
$$

In Gro99, Section 5.34], Gromov called functorial any seminorm (on singular homology of topological spaces) with respect to which every continuous map induces a norm non-increasing morphism. The following result ensures that $\|\cdot\|_{\infty, 0}$ satisfies the obvious analogous of functoriality for seminorms on bounded cohomology of groups:

Lemma 2.1. Let $\psi: \Gamma \rightarrow \Gamma^{\prime}$ be a homomorphism. Then the induced map

$$
\psi^{*}:\left(E H_{b}^{n}\left(\Gamma^{\prime}\right),\|\cdot\|_{\infty, 0}\right) \rightarrow\left(E H_{b}^{n}(\Gamma),\|\cdot\|_{\infty, 0}\right)
$$

is norm non-increasing for every $n \in \mathbb{N}$. 
Proof. Take $\alpha \in E H_{b}^{n}\left(\Gamma^{\prime}\right)$, and let $\varepsilon>0$ be given. Let also $S \subseteq \Gamma$ be an arbitrary finite set. Of course the set $S^{\prime}=\psi(S)$ is finite, so we can find an element $\varphi^{\prime} \in C^{n-1}\left(\Gamma^{\prime}\right)^{\Gamma^{\prime}}$ such that $\left[\delta \varphi^{\prime}\right]=\alpha$ in $E H_{b}^{n}\left(\Gamma^{\prime}\right),\left.\varphi^{\prime}\right|_{S^{\prime}}=0$, and $\left\|\delta \varphi^{\prime}\right\|_{\infty} \leq\|\alpha\|_{\infty, 0}+\varepsilon$. Let now $\varphi=\psi^{*} \varphi^{\prime}$. By construction we have $\left.\varphi\right|_{S}=0$, $[\delta \varphi]=\left[\delta \psi^{*} \varphi^{\prime}\right]=\left[\psi^{*} \delta \varphi^{\prime}\right]=\psi^{*}(\alpha)$, and $\|\delta \varphi\|_{\infty} \leq\left\|\delta \varphi^{\prime}\right\|_{\infty} \leq\|\alpha\|_{\infty, 0}+\varepsilon$. Hence $\left\|\psi^{*}(\alpha)\right\|_{S} \leq\|\alpha\|_{\infty, 0}+\varepsilon$. Due to arbitrariness of $S$ we then have $\left\|\psi^{*}(\alpha)\right\|_{\infty, 0} \leq\|\alpha\|_{\infty, 0}+\varepsilon$, whence the conclusion since $\varepsilon$ is arbitrary.

Corollary 2.2. Let $\psi: \Gamma \rightarrow \Gamma^{\prime}$ be a surjective homomorphism with amenable kernel. Then $\psi^{*}: H_{b}^{n}\left(\Gamma^{\prime}\right) \rightarrow H_{b}^{n}(\Gamma)$ induces an injection

$$
N_{0}^{n}\left(\Gamma^{\prime}\right) \hookrightarrow N_{0}^{n}(\Gamma)
$$

for every $n \in \mathbb{N}$.

Proof. It is well known that an epimorphism with amenable kernel induces an isomorphism in bounded cohomology (see e.g. Gro82, Iva87]), so the conclusion follows from Lemma 2.1.

Question 2.3. Let $\psi: \Gamma \rightarrow \Gamma^{\prime}$ be a surjective homomorphism with amenable kernel. Then the isomorphism $\psi^{*}$ induced by $\psi$ on bounded cohomology is isometric with respect to Gromov's seminorm (see e.g. Gro82, Iva87]). Is it true that $\psi^{*}$ also preserves the seminorm $\|\cdot\|_{\infty, 0}$ on exact bounded cohomology? Or could the seminorm $\|\cdot\|_{\infty, 0}$ be used to distinguish the (exact) bounded cohomology of $\Gamma$ from the (exact) bounded cohomology of $\Gamma^{\prime}$ (as seminormed spaces)?

The norm $\|\cdot\|_{\infty, 0}$ is only interesting in degrees strictly bigger than 2 :

Lemma 2.4. For each non-zero $\alpha$ in $H_{b}^{2}(\Gamma),\|\alpha\|_{\infty, 0}=\infty$.

Proof. Let $C_{n}^{\ell^{1}}(\Gamma)_{\Gamma}$ be the metric completion of $C_{n}(\Gamma)_{\Gamma}$ with respect to the $\ell^{1}$-norm. Being bounded, the differential $\partial_{n}: C_{n}(\Gamma)_{\Gamma} \rightarrow C_{n-1}(\Gamma)_{\Gamma}$ extends to $\ell^{1}$-chains, thus defining a complex whose homology is denoted by $H_{*}^{\ell_{1}}(\Gamma)$. The pairing between $H_{b}^{n}(\Gamma)$ and $H_{n}(\Gamma)$ extends to a pairing between $H_{b}^{n}(\Gamma)$ and $H_{n}^{\ell_{1}}(\Gamma)$. By [MM85, Theorem 2.3 and Corollary 2.7], this pairing induces an isomorphism between $H_{b}^{2}(\Gamma)$ and the dual of $H_{2}^{\ell_{1}}(\Gamma)$. Therefore, it is sufficient to show that if $\alpha \in H_{b}^{2}(\Gamma)$ is any element with $\|\alpha\|_{\infty, 0}=M<\infty$, then $\alpha$ vanishes on every class in $H_{2}^{\ell_{1}}(\Gamma)$. So let $\beta \in C_{2}^{\ell_{1}}(\Gamma)$ be an $\ell^{1}$-cycle, and let $\varepsilon$ be given. We can find a decomposition $\beta=\beta_{1}+\beta_{2}$ such that $\left\|\beta_{2}\right\|_{1}<\varepsilon$ and $\beta_{1}$ is supported on a finite set $S \subseteq \Gamma$. Since $\|\alpha\|_{\infty, 0} \geq\|\alpha\|_{S}$, we can find a representative $a$ of $\alpha$ vanishing on $S$ with Gromov norm smaller than $M+1$. This implies

$$
\langle\alpha,[\beta]\rangle=\left\langle a, \beta_{1}+\beta_{2}\right\rangle=\left\langle a, \beta_{2}\right\rangle \leq \varepsilon(M+1) .
$$

By the arbitrariness of $\varepsilon$, this implies that $\langle\alpha,[\beta]\rangle=0$, as desired.

The rest of the section is devoted to the proof of Theorem 4. We first describe an easy characterization of the seminorm $\|\cdot\|_{\infty, 0}$ defined in the introduction. 
Definition 2.5. An exhaustion of $\Gamma$ is a sequence $\left(S_{i}\right)_{i \in \mathbb{N}}$ of finite subsets $S_{i} \subseteq \Gamma$ such that $S_{i} \subseteq S_{i+1}$ for every $i \in \mathbb{N}$ and $\bigcup_{i \in \mathbb{N}} S_{i}=\Gamma$.

The following criterion is easily verified and very useful in the applications:

Lemma 2.6. Let $\alpha \in E H_{b}^{n+1}(\Gamma)$, and let $\left(S_{i}\right)_{i \in \mathbb{N}}$ be a fixed exhaustion of $\Gamma$. Then for every sequence of elements $\varphi_{i} \in \mathrm{QZ}^{n}(\Gamma)^{\Gamma}, i \in \mathbb{N}$, such that

(1) $\left[\delta \varphi_{i}\right]=\alpha$ for every $i \in \mathbb{N}$,

(2) $\left.\varphi_{i}\right|_{S_{i}}=0$ for every $i \in \mathbb{N}$,

we have

$$
\|\alpha\|_{\infty, 0} \leq \liminf _{i \rightarrow \infty}\left\|\delta \varphi_{i}\right\|_{\infty}
$$

Moreover, one can choose elements $\varphi_{i} \in \mathrm{QZ}^{n}(\Gamma)^{\Gamma}, i \in \mathbb{N}$, satisfying conditions (1) and (2) in such a way that

$$
\|\alpha\|_{\infty, 0}=\lim _{i \rightarrow \infty}\left\|\delta \varphi_{i}\right\|_{\infty} .
$$

2.2. Extension of quasi-cocycles from hyperbolically embedded subgroups. Let us now suppose that $H$ is a hyperbolically embedded subgroup of $\Gamma$, and recall that

$$
r^{*}: H_{b}^{*}(\Gamma) \rightarrow H_{b}^{*}(H)
$$

is the restriction map induced by the inclusion of $H$ in $\Gamma$. We can now proceed with the proof of Theorem 4 , which states that $r^{n+1}$ is an undistorted surjection for every $n \geq 1$, provided that we endow both $E H_{b}^{n+1}(\Gamma)$ and $E H_{b}^{n+1}(H)$ with the $\|\cdot\|_{\infty, 0}$-seminorm (and that $H^{n}(H)$ is finitedimensional). The key ingredient for our argument will be an extension result for quasi-cocycles proved in FPS15.

We first need to introduce the notion of small simplex in $H$. Such notion depends on the geometry of the embedding of $H$ in $\Gamma$. However, for our purposes it is sufficient to know that we can single out a particular finite subset $\bar{S}_{0}$ of $H$ with the property that an element $\bar{h} \in H^{n+1}$ is small if and only if $\bar{h} \in \bar{S}_{0}^{n+1} \subseteq H^{n+1}$ (see [FPS15, Definition 4.7]). In particular, the number of small simplices in $H$ is finite, so for every cochain $\varphi \in C^{n}(H)$ the finite number

$$
K(\varphi)=\max \left\{|\varphi(\bar{h})|, \bar{h} \subseteq H^{n+1} \text { small }\right\}
$$

is well defined.

Now we have the following extension operator for quasi-cocycles on $H$ :

Theorem 2.7 ([FPS15, Theorem 4.1]). There exists a linear map

$$
\Theta^{n}: C_{\text {alt }}^{n}(H)^{H} \rightarrow C_{\text {alt }}^{n}(\Gamma)^{\Gamma}
$$

such that the following conditions hold for every $\varphi \in C_{\mathrm{alt}}^{n}(H)^{H}$ :

(1) $\sup _{\bar{h} \in H^{n+1}}\left|\Theta^{n}(\varphi)(\bar{h})-\varphi(\bar{h})\right| \leq K(\varphi)$;

(2) if $n \geq 2$ then $\left\|\Theta^{n}(\varphi)\right\|_{\infty} \leq n(n+1) \cdot\|\varphi\|_{\infty}$;

(3) $\left\|\delta \Theta^{n}(\varphi)-\Theta^{n+1}(\delta \varphi)\right\|_{\infty} \leq 2(n+1)(n+2) K(\varphi)$. 
Theorem 4 would readily follow from Theorem 2.7 if we could get rid of the additive error $2(n+1)(n+2) K(\varphi)$ described in (3) when estimating the defect of the extension in terms of the defect of the original quasi-cocycle. The following remark shows that this is not possible in general.

Remark 2.8. Let $M$ be an orientable complete finite-volume hyperbolic 3-manifold with one cusp $C$. If $\Gamma=\pi_{1}(M)$ and $H<\Gamma$ is the subgroup corresponding to $C$, then the pair $(\Gamma, H)$ is relatively hyperbolic. In particular, $H$ is hyperbolically embedded in $\Gamma$. Moreover, $H$ is isomorphic to $\mathbb{Z} \oplus \mathbb{Z}$, whence amenable, and the inclusion $C \hookrightarrow M$ induces a non-injective map $H_{2}(C) \rightarrow H_{2}(M)$ in homology. Therefore, [FPS15, Proposition 7.3] shows that there is a genuine cocycle $\varphi \in Z^{2}(H)^{H}$ such that $\left[\delta \Theta^{n}(\varphi)\right]$ is not null in $E H_{b}^{3}(\Gamma)$. In particular, the defect of $\Theta^{2}(\varphi)$ cannot be zero, whereas the defect of $\varphi$ vanishes. This shows that there cannot be any linear bound of the defect of $\Theta^{2}(\varphi)$ in terms of the defect of $\varphi$.

2.3. Proof of Theorem 4. We are now ready to prove Theorem 4. Since in degree 2 the norm $\|\cdot\|_{\infty, 0}$ is infinite on every non-trivial element (see Lemma 2.4), the map $r^{2}: E H_{b}^{2}(G) \rightarrow E H_{b}^{2}(H)$ is obviously undistorted. Therefore, Theorem 4 follows from the following:

Theorem 2.9. Let $n \geq 2$ and assume that $H^{n}(H)$ is finite dimensional. For every $\alpha \in E H_{b}^{n+1}(H)$ there exists $\beta \in E H_{b}^{n+1}(\Gamma)$ such that $r^{n+1}(\beta)=\alpha$ and

$$
\|\beta\|_{\infty, 0} \leq n(n+1)\|\alpha\|_{\infty, 0}
$$

The difficulty in the proof arises from the fact that Theorem 2.7 does not actually give a map $E H_{b}^{n}(H) \rightarrow E H_{b}^{n}(\Gamma)$, but just a map at the level of quasi-cocycles.

Proof. Of course if $\alpha=0$ there is nothing to prove, so we may suppose $\alpha \neq 0$.

Let $\left(S_{i}^{\prime}\right)_{i \in \mathbb{N}}$ be any exhaustion of $\Gamma$. It readily follows from the construction of the map $\Theta^{n}$ in FPS15 that, for every $i \in \mathbb{N}$, there exists a finite subset $S_{i}^{\prime \prime}$ of $H$ such that $\Theta^{n}(\varphi)$ vanishes on $S_{i}^{\prime}$ whenever $\varphi$ vanishes on $\left(S_{i}^{\prime \prime}\right)^{n+1}$. Indeed, if $\bar{g} \in \Gamma^{n+1}$, then

$$
\Theta^{n}(\varphi)(\bar{g})=\sum_{B \in \mathcal{B}} \varphi_{B}^{\prime}\left(\operatorname{tr}_{n}^{B}(\bar{g})\right)
$$

where $B$ varies over all the left cosets of $H$ in $\Gamma, \operatorname{tr}_{n}^{B}(\bar{g})$ is a sort of weighted projection of $\bar{g}$ into $B$ (see [FPS15, Definition 4.5]), and $\varphi^{\prime}$ is obtained from $\varphi$ via the left translation by an element of $B$ (after setting $\varphi=0$ on small simplices contained in $H$ ). It is proved in [FPS15, Theorem 5.1] that the sum in the above definition is finite (i.e. $\varphi_{B}^{\prime}\left(\operatorname{tr}_{n}^{B}(\bar{g})\right)=0$ for all but a finite number of cosets $B$ ), and it readily follows from the definition of $\operatorname{tr}_{n}^{B}$ that $\operatorname{tr}_{n}^{B}(\bar{g})$ is supported on a finite number of simplices in $B$ for every $\bar{g} \in \Gamma^{n+1}$, $B \in \mathcal{B}$. Therefore, we can choose as $S_{i}^{\prime \prime}$ the finite set given by the union of 
the translates in $H$ of the simplices in the support of $\operatorname{tr}_{n}^{B}(\bar{g})$, as $\bar{g}$ varies in $S_{i}^{\prime}$ and $B$ varies among the cosets such that $\operatorname{tr}_{n}^{B}(\bar{g}) \neq 0$.

Moreover, we can suppose that $S_{i}^{\prime \prime} \subseteq S_{i+1}^{\prime \prime}$ for every $i \in \mathbb{N}$ and that $\bigcup_{i \in \mathbb{N}} S_{i}^{\prime \prime}=H$. We now define an exhaustion $\left(S_{i}\right)_{i \in \mathbb{N}}$ of $H$ by setting

$$
S_{i}=S_{i}^{\prime \prime} \cup \bar{S}_{0}, \quad i \in \mathbb{N}
$$

(recall that $\bar{S}_{0}$ is the set of vertices of small simplices in $H$ ).

By Lemma 2.6, there exists a sequence $\varphi_{i}$ of invariant quasi-cocycles on $H$ such that the following conditions hold: for every $i \in \mathbb{N},\left[\delta \varphi_{i}\right]=\alpha$ and $\varphi_{i}(\bar{s})=0$ for every $\bar{s} \in S_{i}^{n+1}$; moreover,

$$
\lim _{i \rightarrow \infty} \operatorname{def}\left(\varphi_{i}\right)=\|\alpha\|_{\infty, 0}
$$

Since alternation does not increase the defect of a quasi-cocycle and does not alter the bounded class of its differential, we can also assume that each $\varphi_{i}$ belongs to $\mathrm{QZ}_{\mathrm{alt}}^{n}(H)^{H}$.

Let us now set $\psi_{i}=\Theta^{n}\left(\varphi_{i}\right) \in C_{\text {alt }}^{n}(\Gamma)^{\Gamma}$. Since $\bar{S}_{0} \subseteq S_{i}$, for every $i \in \mathbb{N}$ we have $K\left(\varphi_{i}\right)=0$, so Theorem 2.7 implies that

$$
\left.\left\|\delta \psi_{i}\right\|_{\infty}=\| \delta \Theta^{n}\left(\varphi_{i}\right)\right)\left\|_{\infty}=\right\| \Theta^{n+1}\left(\delta \varphi_{i}\right)\left\|_{\infty} \leq n(n+1) \cdot\right\| \delta \varphi_{i} \|_{\infty}=n(n+1) \operatorname{def}\left(\varphi_{i}\right) .
$$

In particular, $\psi_{i}$ is an alternating quasi-cocycle with $\operatorname{def}\left(\psi_{i}\right) \leq n(n+$ 1) $\operatorname{def}\left(\varphi_{i}\right)$. By construction we have $\left.\psi_{i}\right|_{S_{i}^{\prime}}=0$, so the differential of $\psi_{i}$ defines a class $\beta_{i} \in E H_{b}^{n+1}(\Gamma)$ such that

$$
\left\|\beta_{i}\right\|_{S_{i}^{\prime}} \leq n(n+1) \operatorname{def}\left(\varphi_{i}\right) .
$$

Moreover, since $K\left(\varphi_{i}\right)=0$, from Theorem 2.7 (1) we deduce that the restriction of $\psi_{i}$ to $H^{n+1}$ coincides with $\varphi_{i}$, so that $r^{n+1}\left(\beta_{i}\right)=\alpha$ for every $i \in \mathbb{N}$.

We are now going to prove the following:

Claim: The $\beta_{i}$ all belong to a finite-dimensional affine subspace of $E H_{b}^{n+1}(\Gamma)$.

To this aim observe that, since $\delta \circ \delta=0$, for every element $c \in C_{\text {alt }}^{n-1}(H)^{H}$ we have

$$
\delta\left(\Theta^{n}(\delta c)\right)=\delta\left(\Theta^{n}(\delta c)-\delta \Theta^{n-1}(c)\right) .
$$

Therefore, by Theorem 2.7 (3), the cochain $\delta\left(\Theta^{n}(\delta c)\right)$ is the coboundary of an invariant bounded cochain, so that it defines the trivial element of $E H_{b}^{n+1}(\Gamma)$. In other words, the map

$$
Z_{\text {alt }}^{n}(H)^{H} \rightarrow E H_{b}^{n+1}(\Gamma), \quad z \mapsto\left[\delta\left(\Theta^{n}(z)\right)\right]
$$

induces a well-defined map

$$
j: H^{n}(H) \rightarrow E H_{b}^{n+1}(\Gamma) .
$$

Since $H^{n}(H)$ is a finite-dimensional vector space, in order to prove the claim it suffices to show that $\beta_{i}-\beta_{j} \in j\left(H^{n}(H)\right)$ for every $i, j \in \mathbb{N}$. Indeed, since $\left[\delta \varphi_{i}\right]=\left[\delta \varphi_{j}\right]$ in $E H_{b}^{n+1}(H)$, we have $\varphi_{i}-\varphi_{j}=b+z$, where $b \in$ 
$C_{b, \text { alt }}^{n}(H)^{H}$ is bounded and $z \in Z_{\text {alt }}^{n}(H)^{H}$ is a genuine cocycle. Since $n \geq 2$, by Theorem 2.7 (2) the cochain $\Theta^{n}(b)$ is bounded, hence

$\beta_{i}-\beta_{j}=\left[\delta \Theta^{n}\left(\varphi_{i}\right)-\delta \Theta^{n}\left(\varphi_{j}\right)\right]=\left[\delta \Theta^{n}(b)+\delta \Theta^{n}(z)\right]=\left[\delta \Theta^{n}(z)\right] \in j\left(H^{n}(H)\right)$, and this proves our claim.

Let now $V$ be the subspace of $E H_{b}^{n+1}(\Gamma)$ generated by the $\beta_{i}, i \in \mathbb{N}$, and let us set $W=r^{n+1}(V)=\operatorname{Span}\langle\alpha\rangle \subseteq E H_{b}^{n+1}(H)$. Assume first that there exists $\beta \in V \cap N_{0}^{n+1}(\Gamma)$ such that $r^{n+1}(\beta) \neq 0$. In this case $r^{n+1}(\beta)$ is a non-zero multiple of $\alpha$, so up to rescaling we may suppose $r^{n+1}(\beta)=\alpha$. We have thus found an element $\beta$ in the preimage of $\alpha$ with vanishing $\|\cdot\|_{\infty, 0^{-}}$ seminorm, and this certainly implies that $\|\beta\|_{\infty, 0} \leq n(n+1)\|\alpha\|_{\infty, 0}$, whence the conclusion.

We may then suppose that $V \cap N_{0}^{n+1}(\Gamma)$ is contained in $\operatorname{ker} r^{n+1}$. In this case we first observe that, since $\|\cdot\|_{S_{i}^{\prime}} \leq\|\cdot\|_{S_{i+1}^{\prime}}$ and $\|\cdot\|_{\infty, 0}=\sup _{i \in \mathbb{N}}\|\cdot\|_{S_{i}^{\prime}}$, the subspaces $M_{i}=\left\{\beta \in V \mid\|\beta\|_{S_{i}^{\prime}}=0\right\}$ satisfy $M_{i} \supseteq M_{i+1}$ and $\bigcap_{i \in \mathbb{N}} M_{i}=$ $V \cap N_{0}^{n+1}(\Gamma)$. Since $V$ is finite-dimensional, this implies in turn that there is $i_{0} \in \mathbb{N}$ such that $V \cap N_{0}^{n+1}(\Gamma)=M_{i}$ for every $i \geq i_{0}$. Moreover, if we denote by $B_{i}$ the subspace of $V$ spanned by $\left\{\beta_{j}, j \geq i\right\}$, then $B_{i} \neq\{0\}$ and $B_{i+1} \subseteq B_{i}$ for every $i \in \mathbb{N}$. Using again that $V$ is finite-dimensional, we obtain that, up to increasing $i_{0}$, we may suppose that $B_{i}=B_{i+1}=B$ for every $i \geq i_{0}$. By definition, for every $i \geq i_{0}$ the subspace $B$ is spanned by elements with finite $\|\cdot\|_{S_{i}^{\prime} \text {-seminorm, and this implies that the seminorm }}$ $\|\cdot\|_{S_{i}^{\prime}}$ is finite on $B$ for every $i \geq i_{0}$.

Let us now define the quotient space $\bar{V}=V /\left(V \cap N_{0}^{n+1}(\Gamma)\right)$ and let us set $\bar{B}=B /\left(B \cap N_{0}^{n+1}(\Gamma)\right) \subseteq \bar{V}$. For every $i \geq i_{0}$, the seminorm $\|\cdot\|_{S_{i}^{\prime}}$ induces a genuine finite and non-degenerate norm on $\bar{B}$, which will still be denoted by $\|\cdot\|_{S_{i}^{\prime}}$. Let us denote by $\bar{\beta}_{i}$ the image of $\beta_{i}$ in $\bar{V}$. Then $\bar{\beta}_{i} \in \bar{B}$ for every $i \geq i_{0}$, and for every $j \geq i_{0}$ we have

$\limsup _{i \rightarrow \infty}\left\|\bar{\beta}_{i}\right\|_{S_{j}^{\prime}} \leq \limsup _{i \rightarrow \infty}\left\|\beta_{i}\right\|_{S_{j}^{\prime}} \leq n(n+1) \lim _{i \rightarrow \infty} \operatorname{def}\left(\varphi_{i}\right)=n(n+1)\|\alpha\|_{\infty, 0}$.

In particular, the $\bar{\beta}_{i}$ are definitively contained in a bounded subset in the finite-dimensional normed space $\left(\bar{B},\|\cdot\|_{S_{i_{0}}^{\prime}}\right)$, and up to passing to a subsequence we can suppose that $\lim _{i \rightarrow \infty} \bar{\beta}_{i}=\bar{\beta}$ in $\left(\bar{B},\|\cdot\|_{S_{i_{0}}^{\prime}}\right)$ for some $\bar{\beta} \in \bar{B}$. Observe now that, being genuine norms on the same finite-dimensional space $\bar{B}$, the norms $\|\cdot\|_{S_{j}^{\prime}}, j \geq i_{0}$, are all equivalent, so $\lim _{i \rightarrow \infty} \bar{\beta}_{i}=\bar{\beta}$ with respect to any norm $\|\cdot\|_{S_{j}^{\prime}}, j \geq i_{0}$. Therefore, thanks to (11) we have

$$
\|\bar{\beta}\|_{S_{j}^{\prime}} \leq n(n+1)\|\alpha\|_{\infty, 0}
$$

for every $j \geq i_{0}$, hence for every $j \in \mathbb{N}$.

Let now $\beta \in V$ be any representative of $\bar{\beta}$. Using (1) we may deduce that

$$
\|\beta\|_{S_{j}^{\prime}}=\|\bar{\beta}\|_{S_{j}^{\prime}} \leq n(n+1)\|\alpha\|_{\infty, 0}
$$


for every $j \geq i_{0}$, and this implies in turn that $\|\beta\|_{\infty, 0} \leq n(n+1)\|\alpha\|_{\infty, 0}$ thanks to Lemma 2.6. Therefore, in order to conclude it suffices to show that $r^{n+1}(\beta)=\alpha$. By construction, the map $r^{n+1}$ induces a map $\bar{r}^{n+1}: \bar{V} \rightarrow$ $W$ such that $\bar{r}^{n+1}(\bar{\beta})=r^{n+1}(\beta)$. If we endow $W$ with its natural Euclidean topology (recall that $W$ is linearly isomorphic to $\mathbb{R}$ ), then the map $\bar{r}^{n+1}: \bar{V} \rightarrow W$, begin linear with a finite-dimensional domain, is continuous with respect to any norm on $\bar{V}$. We thus get

$$
r^{n+1}(\beta)=\bar{r}^{n+1}(\bar{\beta})=\bar{r}^{n+1}\left(\lim _{i \rightarrow \infty} \bar{\beta}_{i}\right)=\lim _{i \rightarrow \infty} r^{n+1}\left(\beta_{i}\right)=\lim _{i \rightarrow \infty} \alpha=\alpha .
$$

This concludes the proof.

2.4. Zero-norm subspaces for acylindrically hyperbolic group. We will now make use of the following fundamental result about acylindrically hyperbolic groups:

Theorem 2.10 (Theorem 2.24 of [DG016]). Let $\Gamma$ be an acylindrically hyperbolic group. Then there exists a hyperbolically embedded subgroup $H$ of $\Gamma$ such that $H$ is isomorphic to $F_{2} \times K$, where $K$ is finite.

The following result shows that Theorem 1 can now be reduced to Theorem 4 and Theorem 2 :

Corollary 2.11. Let $\Gamma$ be an acylindrically hyperbolic group. Then $\operatorname{dim} N_{0}^{n}(\Gamma) \geq$ $\operatorname{dim} N_{0}^{n}\left(F_{2}\right)$ for every $n \in \mathbb{N}$.

Proof. Let $H$ be the hyperbolically embedded subgroup of $\Gamma$ provided by Theorem 2.10, and observe that $H$ surjects onto $F_{2}$ via an epimorphism with finite (whence, amenable) kernel. By Corollary 2.2 we have $\operatorname{dim} N_{0}^{n}\left(F_{2}\right) \leq$ $\operatorname{dim} N_{0}^{n}(H)$, while Theorem 4 ensures that $\operatorname{dim} N_{0}^{n}(H) \leq \operatorname{dim} N_{0}^{n}(\Gamma)$. The conclusion follows.

\section{Relatively hyperbolic GRoups, Cusped SPACES AND BICOMBINGS}

In this section we collect some results about relatively hyperbolic groups that will be useful in the sequel. As described in the introduction, we are going to exhibit non-trivial quasi-cocycles on the free group $F_{2}$ by constructing a combinatorial version of (the primitive) of the volume form on a suitably chosen hyperbolic 3-manifold. By Milnor-Svarc Lemma, the fundamental group of any closed hyperbolic 3-manifold provides a discrete approximation of hyperbolic 3-space. On the other hand, in order to get a quasi-isometric copy of hyperbolic 3-space out of the fundamental group $\Gamma$ of a cusped 3 manifold we need to glue to the Cayley graph of $\Gamma$ an equivariant collection of horoballs. We now briefly describe this procedure, closely following [GM08].

We will only consider simplicial graphs, i.e. graphs without loops and without multiple edges between the same endpoints. Every graph $G$ will be endowed with the path-metric $d_{G}$ induced by giving unitary length to every edge. The set of vertices of $G$ will be denoted by $G^{(0)}$. Following GM08, 
Definition 3.12], we define the (combinatorial) horoball $\mathcal{H}(G)$ based on $G$ as follows. The vertex set of $\mathcal{H}(G)$ is given by $G^{(0)} \times \mathbb{N}$, and two vertices $(g, n)$ and $\left(g^{\prime}, n^{\prime}\right)$ are joined by an edge if and only if one of the following conditions holds:

- either $\left|n-n^{\prime}\right|=1$ and $g=g^{\prime}$,

- or $n=n^{\prime}, g \neq g^{\prime}$ and $d_{G}\left(g, g^{\prime}\right) \leq 2^{n}$.

Let us now fix a finitely generated group $\Gamma$ with a distinguished 11 finitely generated subgroup $H$. We choose a symmetric finite generating set $S$ for $\Gamma$ containing a generating set for $H$, and we denote by Cay $(\Gamma, S)$ the associated Cayley graph, i.e. the graph having $\Gamma$ as the set of vertices, and such that two elements $g, g^{\prime} \in \Gamma=\operatorname{Cay}(\Gamma, S)^{(0)}$ are joined by a single edge if and only if $g^{-1} g^{\prime} \in S$. Observe that the full subgraph of Cay $(\Gamma, S)$ with vertices in $H$ coincides with the Cayley graph of $H$ with respect to the generating set $S \cap H$. The left translation by $g \in \Gamma$ induces an isomorphism between $\operatorname{Cay}(H, S \cap H)$ and the full subgraph of $\operatorname{Cay}(\Gamma, S)$ with vertices in $g H$, which, in particular, is connected. We denote by $\mathcal{H}_{g H}$ the combinatorial horoball based on such subgraph, and we identify the full subgraph of $\mathcal{H}_{g H}$ with vertices in $g H \times\{0\}$ with the full subgraph of $\operatorname{Cay}(\Gamma, S)$ with vertices in $g H$.

Definition 3.1 (GM08]). The cusped graph $X$ associated to the pair $(\Gamma, H)$ (and to a finite generating set $S$ as above) is the graph obtained by gluing a combinatorial horoball $\mathcal{H}_{g H}$ to $\operatorname{Cay}(\Gamma, S)$ for every left coset $g H$ of $H$, via the identification of the full subgraph of $\mathcal{H}_{g H}$ with vertices in $g H \times\{0\}$ with the full subgraph of $\operatorname{Cay}(\Gamma, S)$ with vertices in $g H$.

Remark 3.2. For ease of notation, we often do not distinguish between $X$ and its vertex set.

The relative hyperbolicity of the pair $(\Gamma, H)$ is encoded by the geometry of the cusped graph $X$ as follows:

Theorem 3.3 (GM08, Theorem 3.25]). The pair $(\Gamma, H)$ is relatively hyperbolic if and only if the cusped graph $X$ is Gromov hyperbolic.

Remark 3.4. Indeed, there is a slight difference between our definition of cusped graph and Groves-Manning's one, in that our cusped graphs are necessarily simplicial, whereas Groves and Manning explicitly allow multiple edges in their definition. We avoid double edges because it will be convenient to consider a cusped graph as contained in every Rips complex over it. However, in our applications we will be dealing only with torsion-free groups, for which our definitions precisely coincide with the ones in GM08.

We have that $X^{(0)}$ is in canonical one-to-one correspondence with $\Gamma \times \mathbb{N}$ : this holds because we are dealing with the simple case of a pair $(\Gamma, H)$

\footnotetext{
${ }^{1}$ The constructions and the results that we are going to recall below also hold for groups with a family of distinguished subgroups, but the case of a single subgroup is slightly easier and sufficient to our purposes.
} 
where $H$ is a single subgroup. Henceforth we will tacitly make use of this identification, and denote vertices of $X$ by pairs in $\Gamma \times \mathbb{N}$. Following [GM08], we define the depth function

$$
D: X^{(0)} \rightarrow \mathbb{N}, \quad D(g, n)=n .
$$

For every horoball $\mathcal{H} \subseteq X$ and every $n \in \mathbb{N}$, we define the $n$-horoball $\mathcal{H}_{n} \subseteq X$ associated with $\mathcal{H}$ as the full subgraph of $X$ with vertices in $\mathcal{H}_{n}=D^{-1}([n,+\infty)) \cap \mathcal{H}$. If a cusped graph $X$ is $\delta$-hyperbolic and $C>\delta$, then $C$-horoballs are convex in $X$ [GM08, Lemma 3.26].

3.1. A quasi-geodesic bicombing. We keep notation from the previous section, so that $X$ is the cusped graph associated to a relatively hyperbolic pair $(\Gamma, H)$. Recall from Section 2 that $C_{i}^{\Delta}(X)_{\text {red }}$ denotes the space of reduced simplicial $i$-chains over $X$. A (homological) bicombing $Q: X \times X \rightarrow$ $C_{1}^{\Delta}(X)_{\text {red }}$ is a map such that

$$
\partial Q\left(x_{0}, x_{1}\right)=x_{1}-x_{0}
$$

A bicombing is antisymmetric if $Q\left(x_{0}, x_{1}\right)=-Q\left(x_{1}, x_{0}\right)$ for every $\left(x_{0}, x_{1}\right) \in$ $X^{2}$, and $S$-quasi-geodesic if there exists $S>0$ such that $\operatorname{Supp}\left(Q\left(x_{0}, x_{1}\right)\right)$ is contained in the $S$-neighborhood $\mathcal{N}_{S}(\gamma)$ of any geodesic $\gamma$ joining $x_{0}$ with $x_{1}$ (in GM08, there is the additional requirement that the norm of $Q\left(x_{0}, x_{1}\right)$ be bounded above by $S \cdot d\left(x_{0}, x_{1}\right)$; we will never need this in our argument). Moreover, $Q$ is equivariant if $Q\left(g\left(x_{0}\right), g\left(x_{1}\right)\right)=g \cdot Q\left(x_{0}, x_{1}\right)$ for every $\left(x_{0}, x_{1}\right) \in X^{2}, g \in \Gamma$.

Let $\sigma$ be a 1-simplex in $X$. We define the maximal and the minimal depth of $\sigma$ as follows: $\max D(\sigma)=\max \{D(v), v \in \operatorname{Supp}(\sigma)\}$, while $\min D(\sigma)=$ $\min \{D(v), v \in \operatorname{Supp}(\sigma)\}$ if there exists a horoball containing all the vertices of $\sigma$, and $\min D(\sigma)=-\infty$ otherwise. Then for any given chain $c \in C_{1}^{\Delta}(X)_{\text {red }}$ we set

$$
\begin{aligned}
\max D(c) & =\max \{D(\sigma), \sigma \text { appears in } c\}=\max \{D(v), v \in \operatorname{Supp}(c)\} \in \mathbb{N}, \\
\min D(c) & =\min \{D(\sigma), \sigma \text { appears in } c\} \in \mathbb{N} \cup\{-\infty\} .
\end{aligned}
$$

The existence of a quasi-geodesic bicombing with good filling properties, as stated in Theorem 3.5, is essentially due to Groves and Manning ([GM08, Section 5] and [GM08, Theorem 6.10]). We fix the same notation as in GM08, i.e. we suppose $\delta \geq 1$ and we set

$$
K=10 \delta, \quad L_{1}=100 k, \quad L_{2}=3 L_{1} .
$$

As a quick guide, in the theorem below properties 112415 are just convenient hypotheses to work with small simplices. Property 3 , combined with properties 7 and 8 , says that a bicombing triangle split into a "shallow" part, $z$, and a part that lies deep into the horoballs, $w$. Both $w$ and $z$ are supported near a corresponding geodesic triangle by 6, and the shallow part has bounded norm by 9 (implying that the bicombing chains cancel out nicely in the shallow part). Also, $z$ is alternating by 10 , 
Theorem 3.5. GM08 If $(\Gamma, H)$ is a relatively hyperbolic pair, then there are positive constants $T_{1}, S_{1}$, an $S_{1}$-quasi-geodesic antisymmetric $\Gamma$-equivariant bicombing $Q$ on $X$, and $\Gamma$-equivariant maps $z, w: X^{3} \rightarrow Z_{1}^{\Delta}(X)_{\text {red }}$ with the following properties:

(1) $Q\left(x_{0}, x_{1}\right)=\left[x_{0}, x_{1}\right]$, if $\left[x_{0}, x_{1}\right]$ is an edge of $X$;

(2) if $x_{0}, x_{1}$ belong to the same 0 -horoball $\mathcal{H}$ and $d_{\mathcal{H}}\left(x_{0}, x_{1}\right)=2$, where $d_{\mathcal{H}}$ is the intrinsic distance on $\mathcal{H}$, then $Q\left(x_{0}, x_{1}\right)=\left[x_{0}, y\right]+\left[y, x_{1}\right]$, where $y$ is a vertex in $\mathcal{H}$; in particular, $\operatorname{Supp}\left(Q\left(x_{0}, x_{1}\right)\right) \subseteq \mathcal{H}$;

(3) $Q\left(x_{0}, x_{1}\right)+Q\left(x_{1}, x_{2}\right)+Q\left(x_{2}, x_{0}\right)=z\left(x_{0}, x_{1}, x_{2}\right)+w\left(x_{0}, x_{1}, x_{2}\right)$;

(4) if $\left[x_{i}, x_{j}\right]$ is an edge of $X$ and $D\left(x_{i}\right)=0$ for every $i, j \in\{0,1,2\}$, then $z\left(x_{0}, x_{1}, x_{2}\right)=\left[x_{0}, x_{1}\right]+\left[x_{1}, x_{2}\right]+\left[x_{2}, x_{0}\right]$ and $w\left(x_{0}, x_{1}, x_{2}\right)=0$;

(5) if $x_{0}, x_{1}, x_{2}$ belong to a 0-horoball $\mathcal{H}$ of $X$ and $d_{\mathcal{H}}\left(x_{i}, x_{j}\right) \leq 2$ for every $i, j \in\{0,1,2\}$, then $\operatorname{Supp}\left(z\left(x_{0}, x_{1}, x_{2}\right)\right)$ is contained in $\mathcal{H} \cap$ $B\left(x_{0}, 3\right)$, where $B\left(x_{0}, 3\right)$ is the ball in $X$ of radius 3 centered at $x_{0}$;

(6) $\operatorname{Supp}\left(z\left(x_{0}, x_{1}, x_{2}\right)\right) \cup \operatorname{Supp}\left(w\left(x_{0}, x_{1}, x_{2}\right)\right) \subseteq \mathcal{N}_{S_{1}}\left(\gamma\left(x_{0}, x_{1}\right) \cup \gamma\left(x_{1}, x_{2}\right) \cup\right.$ $\left.\gamma\left(x_{2}, x_{0}\right)\right)$, where $\gamma\left(x_{i}, x_{j}\right)$ is any geodesic joining $x_{i}$ with $x_{j}$;

(7) $\min D\left(w\left(x_{0}, x_{1}, x_{2}\right)\right) \geq L_{2}$;

(8) $\max D\left(z\left(x_{0}, x_{1}, x_{2}\right)\right) \leq 2 L_{2}$;

(9) $\left\|z\left(x_{0}, x_{1}, x_{2}\right)\right\|_{1} \leq T_{1}$;

(10) $z\left(x_{\tau(0)}, x_{\tau(1)}, x_{\tau(2)}\right)=\operatorname{sgn}(\tau) z\left(x_{0}, x_{1}, x_{2}\right)$ and $w\left(x_{\tau(0)}, x_{\tau(1)}, x_{\tau(2)}\right)=$ $\operatorname{sgn}(\tau) w\left(x_{0}, x_{1}, x_{2}\right)$ for every permutation $\tau$ of $\{0,1,2\}$.

Proof. We just define $Q(x, y)$ as the projection of $q_{x, y}$ in $C_{1}^{\Delta}(X)_{\text {red }}$, where $q_{x, y} \in C_{1}^{\Delta}(X)$ is constructed in GM08, as follows. In [GM08, Lemma 3.27] the authors choose an antisymmetric, $\Gamma$-equivariant geodesic bicombing $\gamma$ with the property that if $x$ and $y$ lie in the same $L$-horoball, where $L>2 \delta$, then $\gamma(x, y)$ consists of at most two vertical paths (of arbitrary length) and a horizontal path of length at most 3. Clearly if $d(x, y)=1$ then $\gamma(x, y)$ is the edge between $x$ and $y$. Moreover, if $x$ and $y$ belong to the same 0 -horoball $\mathcal{H}$ and $d_{\mathcal{H}}(x, y)=2$, then it is readily seen that also $d\left(x_{0}, x_{1}\right)=2$, so that the geodesic $\gamma(x, y)$ may be chosen to be equal to a concatenation $\left[x_{0}, y\right] *\left[y, x_{1}\right]$ for some vertex $y \in \mathcal{H}$.

For each pair of points $x, y$ Groves and Manning select an ordered subset $\mathcal{H}_{x, y}$ of the set $\mathcal{C}_{x, y}^{K}$ of $L_{1}$-horoballs intersecting the $K$-neighborhood of $\gamma(x, y)$ [GM08, Remark 4.2, Theorem 4.12], and they define a preferred path $p_{x, y}$ joining $x$ to $y$, in such a way that $p_{x, y}$ decomposes into the concatenation of minimizing geodesics between the horoballs in $\mathcal{H}_{x, y}$ and one suitably chosen path in each $\mathcal{H}_{x, y}$ GM08, Definition 5.7].

The homological bicombing $q_{x, y}$ is then obtained as follows GM08, Definition 6.4]: one decomposes $p_{x, y}$ as a concatenation of segments in $D^{-1}\left[0, L_{2}\right]$ and in $D^{-1}\left[L_{2}, \infty\right)$ where each segment in $D^{-1}\left[L_{2}, \infty\right)$ is contained in one element of $\mathcal{H}_{x, y}$; then, each segment with endpoints $x_{1}, x_{2}$ in $D^{-1}\left[0, L_{2}\right]$ is replaced by the antisymmetric bicombing $Q_{x_{1}, x_{2}}^{\prime}$ constructed by Mineyev in [Min01, and each segment contained in an $L_{2}$-horoball $\mathcal{H}_{L_{2}}$, where $\mathcal{H} \in$ 
$\mathcal{H}_{x, y}$, is replaced by a path in the same $L_{2}$-horoball consisting of at most two vertical paths and a horizontal path of length 1 GM08, Definition 6.4]. Finally, $q_{x, y}$ is antisymmetrized. Conditions (11) and (2) now follow from the explicit description of $Q$ inside $L_{2}$-horoballs, together with the fact that Mineyev's bicombing $Q_{x_{1}, x_{2}}^{\prime}$ is obtained by antisymmetrizing $p_{x_{1}, x_{2}}=\gamma\left(x_{1}, x_{2}\right)$ if $d\left(x_{1}, x_{2}\right) \leq 10 \delta$. Moreover, GM08, Proposition 6.5] implies that $Q$ is $S_{1}$-geodesic.

Henceforth we denote by $Q$ also the obvious linear extension of $Q$ to linear combinations of pairs, so that

$$
Q\left(\partial\left(x_{0}, x_{1}, x_{2}\right)\right)=Q\left(x_{0}, x_{1}\right)+Q\left(x_{1}, x_{2}\right)+Q\left(x_{2}, x_{0}\right) .
$$

We first define the cycles $z\left(x_{0}, x_{1}, x_{2}\right)$ and $w\left(x_{0}, x_{1}, x_{2}\right)$ in the particular cases described in items (44) and (5). We first suppose that $\left[x_{i}, x_{j}\right]$ is an edge of $X$ and $D\left(x_{i}\right)=0$ for every $i, j \in\{0,1,2\}$. Then we set $z\left(x_{0}, x_{1}, x_{2}\right)=$ $Q\left(\partial\left(x_{0}, x_{1}, x_{2}\right)\right)=\left[x_{0}, x_{1}\right]+\left[x_{1}, x_{2}\right]+\left[x_{2}, x_{3}\right]$ and $w\left(x_{0}, x_{1}, x_{2}\right)=0$, and it is immediate to check that this choice fulfills all the requirements of the statement.

Suppose now $x_{0}, x_{1}, x_{2}$ belong to a 0 -horoball $\mathcal{H}$ of $\mathcal{X}$ and $d_{\mathcal{H}}\left(x_{i}, x_{j}\right) \leq 2$ for every $i, j \in\{0,1,2\}$. By claims (1) and (2), the cycle $Q\left(\partial\left(x_{0}, x_{1}, x_{2}\right)\right)$ is the sum of at most 6 consecutive edges of $\mathcal{H}$, and $x_{0}$ is an endpoint of one of these edges. Therefore, the support of $Q\left(\partial\left(x_{0}, x_{1}, x_{2}\right)\right)$ is contained in $\mathcal{H} \cap B\left(x_{0}, 3\right)$. Let us now distinguish two cases: if $D\left(x_{i}\right) \geq$ $L_{2}+3$ for every $i=0,1,2$, then $\min D\left(\operatorname{Supp}\left(Q\left(\partial\left(x_{0}, x_{1}, x_{2}\right)\right)\right)\right) \geq L_{2}$, and we set $z\left(x_{0}, x_{1}, x_{2}\right)=0, w\left(x_{0}, x_{1}, x_{2}\right)=Q\left(\partial\left(x_{0}, x_{1}, x_{2}\right)\right)$. Otherwise, $\max D\left(\operatorname{Supp}\left(Q\left(\partial\left(x_{0}, x_{1}, x_{2}\right)\right)\right)\right) \leq 2 L_{2}$, and we set $z\left(x_{0}, x_{1}, x_{2}\right)=Q\left(\partial\left(x_{0}, x_{1}, x_{2}\right)\right)$ and $w\left(x_{0}, x_{1}, x_{2}\right)=0$.

Let us now suppose that the triple $\left(x_{0}, x_{1}, x_{2}\right)$ does not fall into the cases described in items (44) and (5). We denote by $\bar{z}\left(x_{0}, x_{1}, x_{2}\right)$ the reduced cycle associated to the cycle $c_{x_{0} x_{1} x_{2}}$ defined in [GM08, Definition 6.8], and we set

$$
\bar{w}\left(x_{0}, x_{1}, x_{2}\right)=Q\left(\partial\left(x_{0}, x_{1}, x_{2}\right)\right)-\bar{z}\left(x_{0}, x_{1}, x_{2}\right),
$$

and

$$
\begin{aligned}
& z\left(x_{0}, x_{1}, x_{2}\right)=\frac{1}{6} \sum_{\tau \in \mathfrak{S}_{3}} \bar{z}\left(x_{\tau(0)}, x_{\tau(1)}, x_{\tau(2)}\right), \\
& w\left(x_{0}, x_{1}, x_{2}\right)=\frac{1}{6} \sum_{\tau \in \mathfrak{S}_{3}} \bar{w}\left(x_{\tau(0)}, x_{\tau(1)}, x_{\tau(2)}\right) .
\end{aligned}
$$

Since $\bar{z}$ is a cycle, then so are $\bar{w}, z$ and $w$. Conditions (3) and (10) follow from the definitions and from the fact that $Q$ is antisymmetric.

Since $z, w$ are obtained from $\bar{z}, \bar{w}$ via alternation, in the proof of items (6) , (7), (8), (9) we can replace $z, w$ with $\bar{z}, \bar{w}$, respectively.

The fact that $\bar{z}, \bar{w}$ satisfy properties (7) and (9) is proved in GM08, Theorem 6.10].

In order to show (6) and (8) we need to describe the construction of $\bar{z}\left(x_{0}, x_{1}, x_{2}\right)$ in more detail. For any triple $\left(x_{0}, x_{1}, x_{2}\right)$ of vertices of $X$, a 
preferred triangle with vertices $x_{0}, x_{1}, x_{2}$ is a map $\psi: \partial \Delta^{2} \rightarrow X$ which takes the vertices and the sides of $\Delta^{2}$ respectively to $x_{0}, x_{1}, x_{2}$ and to the preferred paths $p_{x_{0}, x_{1}}, p_{x_{1}, x_{2}}, p_{x_{2}, x_{0}}$ GM08, Definition 5.28]. A skeletal filling of $\psi$ is a map $\ddot{\psi}: \operatorname{Skel}(\psi) \rightarrow X$ of $\psi$, where $\operatorname{Skel}(\psi)$ is a 1-complex containing suitable subdivisions of the sides of $\Delta^{2}$, and $\ddot{\psi}$ is a continuous map extending $\psi$ GM08, Definition 5.26]. A thick subpicture of $\operatorname{Skel}(\psi)$ is (the quotient of) a subgraph of $\operatorname{Skel}(\psi)$ which is taken by (the map induced by) $\ddot{\psi}$ into the thick part $D^{-1}\left(\left[0, L_{2}\right]\right)$ of $X$ (and which enjoys several additional properties that we do not describe here, see GM08, Definition 5.42]). Finally, $c_{x_{0}, x_{1}, x_{2}}$ is a finite sum of terms of the form $Q(\ddot{\psi}(v), \ddot{\psi}(w))$, where $v, w$ are consecutive vertices of a thick subpicture of $\operatorname{Skel}(\psi)$ (see [GM08, Definition 6.8]).

Let us now prove (8) . The explicit description of $\bar{z}\left(x_{0}, x_{1}, x_{2}\right)$ implies that, in order to bound $\max D\left(\operatorname{Supp}\left(\bar{z}\left(x_{0}, x_{1}, x_{2}\right)\right)\right)=\max D\left(\operatorname{Supp}\left(c_{x_{0}, x_{1}, x_{2}}\right)\right)$, it is sufficient to bound $\max D(\operatorname{Supp}(Q(\ddot{\psi}(v), \ddot{\psi}(w))))$, where $v, w$ are consecutive vertices of a thick subpicture. However, GM08, Proposition 5.43] implies that, if $\gamma$ is any geodesic joining $\ddot{\psi}(v), \ddot{\psi}(w)$, then $\gamma$ does not intersect any $\left(L_{1}+L_{2}\right)$-horoball. Moreover, GM08, Proposition 6.5] implies that $\operatorname{Supp}(Q(\ddot{\psi}(v), \ddot{\psi}(w)))$ is contained in the $(K+25 \delta+9)$-neighborhood of $\gamma$, so that $\max D(\operatorname{Supp}(Q(\ddot{\psi}(v), \ddot{\psi}(w)))) \leq L_{1}+L_{2}+K+25 \delta+9 \leq 2 L_{2}$. This implies (8).

We are finally left to prove (6). We first show that

$$
\operatorname{Supp}\left(\bar{z}\left(x_{0}, x_{1}, x_{2}\right)\right) \subseteq \mathcal{N}_{S_{1}}\left(\gamma\left(x_{0}, x_{1}\right) \cup \gamma\left(x_{1}, x_{2}\right) \cup \gamma\left(x_{2}, x_{0}\right)\right)
$$

for a suitably chosen universal constant $S_{1}$. Indeed, as observed in the first paragraph of the proof of [GM08, Proposition 5.43], if $v, w$ are two consecutive vertices of a thick subpicture of $\operatorname{Skel}(\psi)$, then either $d(\ddot{\psi}(v), \ddot{\psi}(w))=1$ or $v, w$ both lie on the same side of $\partial \Delta^{2}$. In the former case $Q(\ddot{\psi}(v), \ddot{\psi}(w))=$ $[v, w]$, which is supported in the 1-neighborhood of $p_{x_{0}, x_{1}} \cup p_{x_{1}, x_{2}} \cup p_{x_{2}, x_{0}}$, which in turn is supported in the $(K+12 \delta+9)$-neighborhood of $\gamma\left(x_{0}, x_{1}\right) \cup$ $\gamma\left(x_{1}, x_{2}\right) \cup \gamma\left(x_{2}, x_{0}\right)$ for any geodesic $\gamma\left(x_{i}, x_{j}\right)$ between $x_{i}$ and $x_{j}$ (see GM08, Corollary 5.12]). In the latter case, suppose that $\ddot{\psi}(u), \ddot{\psi}(v)$ lie on the preferred path $p_{x_{i}, x_{j}}$. Let $p(\ddot{\psi}(v), \ddot{\psi}(w))$ be the subpath of $p_{x_{i}, x_{j}}$ with endpoints $\ddot{\psi}(v), \ddot{\psi}(w)$, and let $\gamma(\ddot{\psi}(v), \ddot{\psi}(w))$ be any geodesic with the same endpoints. Finally, let $\gamma\left(x_{i}, x_{j}\right)$ be any geodesic joining $x_{i}$ with $x_{j}$. By [GM08, Proposition 6.5], the chain $Q(\ddot{\psi}(v), \ddot{\psi}(w))$ is supported in the $(K+25 \delta+9)$ neighborhood of $\gamma(\ddot{\psi}(v), \ddot{\psi}(w))$. By GM08, Corollary 5.13], $p(\ddot{\psi}(v), \ddot{\psi}(w))$ is a quasi-geodesic with uniformly bounded quasi-geodesicity constants, so by hyperbolicity of $X$ there exists a universal constant $S^{\prime}$ such that the Hausdorff distance between $\gamma(\ddot{\psi}(v), \ddot{\psi}(w))$ and $p(\ddot{\psi}(v), \ddot{\psi}(w))$ is bounded by $S^{\prime}$. Finally, GM08, Corollary 5.12] ensures that $p_{x_{i}, x_{j}}$, whence $p(\ddot{\psi}(v), \ddot{\psi}(w))$, is contained in the $(K+12 \delta+9)$-neighborhood of $\gamma\left(x_{i}, x_{j}\right)$. Summing up, we have that the support of $Q(\ddot{\psi}(v), \ddot{\psi}(w))$ is contained in the $S_{1}$-neighborhood of $\gamma\left(x_{0}, x_{1}\right) \cup \gamma\left(x_{1}, x_{2}\right) \cup \gamma\left(x_{2}, x_{0}\right)$, where $S_{1}=S^{\prime}+2 K+37 \delta+18$. This 
concludes the proof that

$$
\operatorname{Supp}\left(\bar{z}\left(x_{0}, x_{1}, x_{2}\right)\right) \subseteq \mathcal{N}_{S_{1}}\left(\gamma\left(x_{0}, x_{1}\right) \cup \gamma\left(x_{1}, x_{2}\right) \cup \gamma\left(x_{2}, x_{0}\right)\right) .
$$

Recall now from [GM08, Proposition 6.5] that

$$
\begin{aligned}
\operatorname{Supp}\left(Q\left(\partial\left(x_{0}, x_{1}, x_{2}\right)\right)\right) & \subseteq \mathcal{N}_{K+25 \delta+9}\left(\gamma\left(x_{0}, x_{1}\right) \cup \gamma\left(x_{1}, x_{2}\right) \cup \gamma\left(x_{2}, x_{0}\right)\right) \\
& \subseteq \mathcal{N}_{S_{1}}\left(\gamma\left(x_{0}, x_{1}\right) \cup \gamma\left(x_{1}, x_{2}\right) \cup \gamma\left(x_{2}, x_{0}\right)\right),
\end{aligned}
$$

so from

$$
\bar{w}\left(x_{0}, x_{1}, x_{2}\right)=Q\left(\partial\left(x_{0}, x_{1}, x_{2}\right)\right)-\bar{z}\left(x_{0}, x_{1}, x_{2}\right)
$$

we now readily deduce that also

$$
\operatorname{Supp}\left(\bar{w}\left(x_{0}, x_{1}, x_{2}\right)\right) \subseteq \mathcal{N}_{S_{1}}\left(\gamma\left(x_{0}, x_{1}\right) \cup \gamma\left(x_{1}, x_{2}\right) \cup \gamma\left(x_{2}, x_{0}\right)\right) .
$$

This concludes the proof of item (6).

3.2. Rips complexes on cusped graphs. We are now interested in proving some results about fillings of cycles in relatively hyperbolic groups. It is well known that hyperbolic groups may be characterized as those groups which satisfy a linear isoperimetric inequality, and an analogous characterization also holds for relatively hyperbolic groups, provided that fillings are replaced by suitably defined relative fillings. Classical isoperimetric inequalities usually deal with fillings of 1-cycles via 2-chains, and in order to provide group-theoretic definitions of length and area it is usually sufficient to take generators and relations as unitary segments and as tiles of unitary area, respectively. However, in our argument we also need higher dimensional isoperimetric inequalities, which are better stated in the context of higher dimensional complexes. To this aim it is often useful to consider Rips complexes (over augmented Cayley graphs, in our case of interest).

Definition 3.6. Given a graph $G$ and a parameter $1 \leq \kappa \in \mathbb{N}$, the Rips complex $\mathcal{R}_{\kappa}(G)$ on $G$ is the simplicial complex having $G^{(0)}$ as set of vertices, and an $n$-dimensional simplex for every $(n+1)$-tuple of vertices whose diameter in $G$ is at most $\kappa$.

Let now $X$ be the cusped graph associated to the relative hyperbolic pair $(\Gamma, H)$, as in the previous subsections. We fix a constant $\kappa \geq 4 \delta+6$, where $\delta \in \mathbb{N}$ is a hyperbolicity constant for $X$, and we set

$$
\mathcal{X}=\mathcal{R}_{\kappa}(X) \text {. }
$$

It is well known that, for $\kappa \geq 4 \delta+6$, the Rips complex $\mathcal{R}_{\kappa}(G)$ of a $\delta$ hyperbolic graph is contractible (see for example [BH99a, 3.Г.3.23]). Therefore, we have the following:

Proposition 3.7. The simplicial complex $\mathcal{X}$ is contractible.

The notion of horoball easily carries over to $\mathcal{X}$ as follows:

Definition 3.8. An $(n-)$ horoball of $\mathcal{X}$ is a full subcomplex of $\mathcal{X}$ having the same vertices as an $(n-)$ horoball of $X$. 
The maximal and the minimal depth of a chain $c \in C_{n}^{\Delta}(\mathcal{X})_{\text {red }}$ are defined exactly as we did for $X$.

Observe that, since $\kappa \geq 1$, the graph $X$ is naturally a subcomplex of $\mathcal{X}$. We stress the fact that, when we refer to the distance in $\mathcal{X}$, we will always refer to the restriction of the distance of $X$ to the vertices of $\mathcal{X}$ : we will be never interested in defining a metric on the internal part of $i$-simplices of $\mathcal{X}$, $i \geq 1$, or in understanding the path metric associated to the structure of $\mathcal{X}$ as a simplicial complex. In particular, if $A$ is any subcomplex of $\mathcal{X}$, then we denote by $\mathcal{N}_{S}(A)$ the full subcomplex of $\mathcal{X}$ whose vertices lie at distance (in $X)$ at most $S$ from the set of vertices of $A$.

The isometric action of $\Gamma$ on $X$ induces a simplicial action of $\Gamma$ on $\mathcal{X}$. As a consequence, each $C_{n}^{\Delta}(\mathcal{X})_{\text {red }}, n \in \mathbb{N}$, is endowed with the structure of a normed $\Gamma$-module (i.e. a normed space equipped with an isometric $\Gamma$-action).

The first author constructed in [Fra] fillings of cycles in $Z_{k}(\mathcal{X})$ with good properties:

Theorem 3.9 ([Fra, Theorem 5.6]). Let $n, k, S \in \mathbb{N}, k \geq 1$. Then there exists $S^{\prime}=S^{\prime}(n, k, S) \in \mathbb{N}$ such that, for every cycle $a \in Z_{k}^{\Delta}(\mathcal{X})_{\text {red }}$ and every family of geodesic segments $\alpha_{1}, \ldots, \alpha_{n}$ such that $\operatorname{Supp}(a) \subseteq \mathcal{N}_{S}\left(\alpha_{1} \cup\right.$ $\left.\ldots \cup \alpha_{n}\right)$, there exists $b \in C_{k+1}^{\Delta}(\mathcal{X})_{\text {red }}$ with $\partial b=$ a such that

(1) $\operatorname{Supp}(b) \subseteq \mathcal{N}_{S^{\prime}}(\operatorname{Supp}(a))$ (in particular, $\operatorname{Supp}(b) \subseteq \mathcal{N}_{S+S^{\prime}}\left(\alpha_{1} \cup \ldots \cup\right.$ $\left.\alpha_{n}\right)$ ),

(2) $\|b\|_{1} \leq M(n, k, S, \max D(z))\|a\|_{1}$,

(3) if $\operatorname{Supp}(a)$ is contained in a $(2 \delta)$-horoball, then $\operatorname{Supp}(b)$ is contained in the same $(2 \delta)$-horoball.

Definition 3.10. Take $z \in C_{k}^{\Delta}(\mathcal{X})_{\text {red }}$. We say that a chain $a \in C_{k+1}^{\Delta}(\mathcal{X})_{\text {red }}$ is a relative filling of $z$ if

$$
z=\partial a+c
$$

where $c$ is a chain in $C_{k}^{\Delta}(\mathcal{X})_{\text {red }}$ with $\min D(c) \geq 0$ (i.e. each simplex appearing in $c$ is contained in some 0 -horoball).

We will now use Franceschini's result to construct a relative filling of the bicombing $Q$ described above.

Proposition 3.11. There exist constants $T_{2}, T_{3} \in \mathbb{R}$ and a $\Gamma$-equivariant map $\varphi: X^{3} \rightarrow C_{2}^{\Delta}(\mathcal{X})_{\text {red }}$ such that, for any triple $\left(x_{0}, x_{1}, x_{2}\right)$ of vertices in $X:$

(1) if $\left[x_{i}, x_{j}\right]$ is an edge of $X$ and $D\left(x_{i}\right)=0$ for every $i, j \in\{0,1,2\}$, then $\varphi\left(x_{0}, x_{1}, x_{2}\right)=\left[x_{0}, x_{1}, x_{2}\right]$,

(2) if $x_{0}, x_{1}, x_{2}$ belong to a 0-horoball $\mathcal{H}$ of $\mathcal{X}$ and $d_{\mathcal{H}}\left(x_{i}, x_{j}\right) \leq 2$ for every $i, j \in\{0,1,2\}$, then $\operatorname{Supp}\left(\varphi\left(x_{0}, x_{1}, x_{2}\right)\right) \subseteq \mathcal{H}$,

(3) $\left\|\varphi\left(x_{0}, x_{1}, x_{2}\right)\right\|_{1} \leq T_{2}$,

(4) the chain $\varphi\left(\partial\left(x_{0}, x_{1}, x_{2}, x_{3}\right)\right)$ admits a relative filling $B \in C_{3}^{\Delta}(\mathcal{X})_{\text {red }}$ such that $\|B\|_{1} \leq T_{3}$. 
Proof. In order to get an equivariant map, we first define $\varphi$ on a set of representatives for the action of $\Gamma$ on $X^{3}$, and then we extend $\varphi$ equivariantly. Since $\Gamma$ acts by isometries on $X$ and leaves the depth of points invariant, it is clear that this choice is coherent with requirements (1) and (2) of the statement.

Let us fix an element $\left(x_{0}, x_{1}, x_{2}\right)$ in the fixed set of representatives. We set

$$
z=z\left(x_{0}, x_{1}, x_{2}\right), \quad w=w\left(x_{0}, x_{1}, x_{2}\right),
$$

where $z\left(x_{0}, x_{1}, x_{2}\right)$ and $w\left(x_{0}, x_{1}, x_{2}\right)$ are the cycles provided by Theorem 3.5 . We will define $\varphi\left(x_{0}, x_{1}, x_{2}\right)$ as a suitably chosen filling of $z$.

We first take care of the cases described in items (1) and (2). Suppose that $\left[x_{i}, x_{j}\right]$ is an edge of $X$ and $D\left(x_{i}\right)=0$ for every $i, j \in\{0,1,2\}$. By Theorem 3.5 (11), we have $z=\left[x_{0}, x_{1}\right]+\left[x_{1}, x_{2}\right]+\left[x_{2}, x_{0}\right]$, and we just set $\varphi\left(x_{0}, x_{1}, x_{2}\right)=\left[x_{0}, x_{1}, x_{2}\right]$.

Suppose now $x_{0}, x_{1}, x_{2}$ belong to a 0 -horoball $\mathcal{H}$ of $\mathcal{X}$ and $d_{\mathcal{H}}\left(x_{i}, x_{j}\right) \leq 2$ for every $i, j \in\{0,1,2\}$. By claim (5) of Theorem 3.5, if $z=\sum_{i} \lambda_{i}\left[x_{i}^{\prime}, x_{i}^{\prime \prime}\right]$, then $x_{i}^{\prime}, x_{i}^{\prime \prime} \in B\left(x_{0}, 3\right) \cap \mathcal{H}$ for every $i$. Since $\mathcal{X}=\mathcal{R}_{\kappa}(X)$ and $\kappa \geq 4$, this implies that $\left\{x_{0}, x_{i}^{\prime}, x_{i}^{\prime \prime}\right\}$ is the set of vertices of a simplex of $\mathcal{X}$. Therefore, the sum $\varphi\left(x_{0}, x_{1}, x_{2}\right)=\sum_{i} \lambda_{i}\left[x_{0}, x_{i}^{\prime}, x_{i}^{\prime \prime}\right]$ defines an element in $C_{2}^{\Delta}(\mathcal{X})_{\text {red }}$ supported on $\mathcal{H}$ such that $\partial \varphi\left(x_{0}, x_{1}, x_{2}\right)=z$ and $\left\|\varphi\left(x_{0}, x_{1}, x_{2}\right)\right\|_{1}=\|z\|_{1} \leq$ $T_{1}$.

Suppose now that the triple $\left(x_{0}, x_{1}, x_{2}\right)$ does not satisfy the conditions described in items (1) and (2). By Theorem 3.5 (6) $), \operatorname{Supp}(z)$ is contained in the $S_{1}$-neighborhood of the union $\gamma\left(x_{0}, x_{1}\right) \cup \gamma\left(x_{1}, x_{2}\right) \cup \gamma\left(x_{2}, x_{0}\right)$, where $\gamma\left(x_{i}, x_{j}\right)$ is any geodesic joining $x_{i}$ with $x_{j}$ (and $S_{1}$ does not depend on $\left(x_{0}, x_{1}, x_{2}\right)$ ). Moreover, $\max D(z) \leq 2 L_{2}$ and $\|z\|_{1} \leq T_{1}$ because of (8) and (9) of Theorem 3.5. Hence by Theorem 3.9 there exists a chain $\varphi\left(x_{0}, x_{1}, x_{2}\right) \in$ $C_{2}^{\Delta}(\mathcal{X})_{\text {red }}$ such that $\partial \varphi\left(x_{0}, x_{1}, x_{2}\right)=z$ and $\left\|\varphi\left(x_{0}, x_{1}, x_{2}\right)\right\|_{1} \leq T_{2}$, where $T_{2}=M\left(3,1, S_{1}, 2 L_{2}\right) \cdot T_{1}$. This concludes the proof of (1), (2) and (3). Also observe that, if $S_{1}^{\prime}=S^{\prime}\left(3,1, S_{1}\right)+S_{1}$, then by Theorem 3.9 (1)

$$
\operatorname{Supp}\left(\varphi\left(x_{0}, x_{1}, x_{2}\right)\right) \subseteq \mathcal{N}_{S_{1}^{\prime}}\left(\gamma\left(x_{0}, x_{1}\right) \cup \gamma\left(x_{1}, x_{2}\right) \cup \gamma\left(x_{2}, x_{0}\right)\right),
$$

where $\gamma\left(x_{i}, x_{j}\right)$ is any geodesic joining $x_{i}$ with $x_{j}$.

We now construct the relative filling $B$ of $\varphi\left(\partial\left(x_{0}, x_{1}, x_{2}, x_{3}\right)\right)$ required to prove claim (4). Since $\varphi\left(\partial\left(x_{0}, x_{1}, x_{2}, x_{3}\right)\right)$ is not a cycle, we need to find first a chain $c$ supported in the horoballs and satisfying $\partial c=\partial \varphi\left(\partial\left(x_{0}, x_{1}, x_{2}, x_{3}\right)\right)$. For the sake of conciseness, we will denote by $z$ and $w$ also the linear extensions of $z$ and $w$ over linear combinations of triples in $X^{3}$, so that, for example, $z\left(\partial\left(x_{0}, \ldots, x_{3}\right)\right)=\sum_{i=0}^{3}(-1)^{i} z\left(x_{0}, \ldots, \widehat{x}_{i}, \ldots, x_{3}\right)$. Let us fix $\left(x_{0}, \ldots, x_{3}\right) \in X^{4}$. Since $Q \circ \partial=z+w$ and $Q \circ \partial \circ \partial=0$, we have

$$
z\left(\partial\left(x_{0}, \ldots, x_{3}\right)\right)=-w\left(\partial\left(x_{0}, \ldots, x_{3}\right)\right) .
$$

Therefore, claims (7) and (8) of Theorem 3.5] imply that

$$
\max D\left(z\left(\partial\left(x_{0}, \ldots, x_{3}\right)\right)\right) \leq 2 L_{2},
$$


THE ZERO NORM SUBSPACE FOR ACYLINDRICALLY HYPERBOLIC GROUPS 28

$$
\min D\left(z\left(\partial\left(x_{0}, \ldots, x_{3}\right)\right)\right)=\min D\left(w\left(\partial\left(x_{0}, \ldots, x_{3}\right)\right)\right) \geq L_{2} .
$$

Moreover, by Theorem 3.5 (9) we have

$$
\left\|z\left(\partial\left(x_{0}, \ldots, x_{3}\right)\right)\right\|_{1}=\left\|\sum_{i=0}^{3}(-1)^{i} z\left(x_{0}, \ldots, \widehat{x}_{i}, \ldots, x_{3}\right)\right\|_{1} \leq 4 T_{1} .
$$

Also observe that, by Theorem 3.5 (6), we have

$$
\operatorname{Supp}\left(z\left(\partial\left(x_{0}, \ldots, x_{3}\right)\right) \subseteq \mathcal{N}_{S_{1}}\left(\bigcup_{i, j=0}^{3} \gamma\left(x_{i}, x_{j}\right)\right),\right.
$$

where $\gamma\left(x_{i}, x_{j}\right)$ is any fixed geodesic joining $x_{i}$ with $x_{j}$. Since $L_{2} \geq 2 \delta$, Theorem 3.9 implies that there exists a chain $c$ such that, if $T_{1}^{\prime}=4 M\left(6,1, S_{1}, 2 L_{2}\right) T_{1}$ and $S_{1}^{\prime \prime}=S^{\prime}\left(6,1, S_{1}\right)+S_{1}$, then

$$
\begin{gathered}
\partial c=z\left(\partial\left(x_{0}, \ldots, x_{3}\right)\right), \\
\operatorname{Supp}(c) \subseteq \mathcal{N}_{S_{1}^{\prime \prime}}\left(\bigcup_{i, j=0}^{3} \gamma\left(x_{i}, x_{j}\right)\right) \\
\|c\|_{1} \leq M\left(6,1, S_{1}, 2 L_{2}\right) \cdot\left\|z\left(\partial\left(x_{0}, \ldots, x_{3}\right)\right)\right\|_{1} \leq T_{1}^{\prime}, \\
\min D(c) \geq 2 \delta
\end{gathered}
$$

(in particular, each simplex appearing in $c$ is contained in some horoball). Moreover, since $\operatorname{Supp}(c) \subseteq \mathcal{N}_{S_{1}^{\prime \prime}}\left(\operatorname{Supp}\left(z\left(\partial\left(x_{0}, \ldots, x_{3}\right)\right)\right)\right)$, we also have

$$
\max D(c) \leq \max D\left(z\left(\partial\left(x_{0}, \ldots, x_{3}\right)\right)\right)+S_{1}^{\prime \prime} \leq 2 L_{2}+S_{1}^{\prime \prime} .
$$

Let us now consider the chain

$$
a=\varphi\left(\partial\left(x_{0}, x_{1}, x_{2}, x_{3}\right)\right)-c .
$$

By construction, $\partial a=0$, i.e. $a$ is a cycle. If $S_{1}^{\prime \prime \prime}=\max \left\{S_{1}^{\prime}, S_{1}^{\prime \prime}\right\}$, then

$$
\operatorname{Supp}(a) \subseteq \mathcal{N}_{S_{1}^{\prime \prime \prime}}\left(\bigcup_{i, j=0}^{3} \gamma\left(x_{i}, x_{j}\right)\right) \text {. }
$$

Moreover,

$$
\max D(a) \leq \max \left\{D(c), D\left(z\left(\partial\left(x_{0}, \ldots, x_{3}\right)\right)\right)\right\} \leq 2 L_{2}+S_{1}^{\prime \prime} .
$$

Let $B$ be the filling of $a$ provided by Theorem 3.9 , By construction, $\partial B=$ $a=\varphi\left(\partial\left(x_{0}, x_{1}, x_{2}, x_{3}\right)\right)-c$, so $B$ is a relative filling of $\varphi\left(\partial\left(x_{0}, x_{1}, x_{2}, x_{3}\right)\right)$. Moreover,

$$
\begin{aligned}
\|B\|_{1} & \leq M\left(6,2, S_{1}^{\prime \prime \prime}, 2 L_{2}+S_{1}^{\prime \prime}\right)\|a\|_{1} \\
& \leq M\left(6,2, S_{1}^{\prime \prime \prime}, 2 L_{2}+S_{1}^{\prime \prime}\right)\left(\left\|\varphi\left(\partial\left(x_{0}, x_{1}, x_{2}, x_{3}\right)\right)\right\|_{1}+\|c\|_{1}\right) \\
& \leq M\left(6,2, S_{1}^{\prime \prime \prime}, 2 L_{2}+S_{1}^{\prime \prime}\right)\left(4 T_{2}+T_{1}^{\prime}\right) .
\end{aligned}
$$

This concludes the proof. 


\section{Combinatorial volume Forms}

Before going into the proof of Theorem 5, let us fix some notation. Let $\Gamma_{0}=F(a, b)$ be a free group of rank 2 , and let $\psi: \Gamma_{0} \rightarrow \Gamma_{0}$ be a group automorphism induced by a pseudo-Anosov orientation-preserving homeomorphism of a punctured torus. Up to conjugating $\psi$, we may suppose that $\psi([a, b])=[a, b]$. Let $\Gamma=\Gamma_{0} \rtimes_{\psi} \mathbb{Z}$, and denote by $t$ the generator of $\mathbb{Z}<\Gamma$, in such a way that $t g t^{-1}=\psi(g)$ for every $g \in \Gamma_{0}$. Let $H<\Gamma$ be the subgroup generated by $[a, b]$ and $t$, and recall that the pair $(\Gamma, H)$ is relatively hyperbolic. We denote by $X$ the cusped graph associated to the pair $(\Gamma, H)$ and the generating set $S=\{a, b,[a, b], t\}$, and by $\mathcal{X}$ the contractible Rips complex over $X$ defined in the previous section. In fact, we will completely forget the structure of $X$ as a graph, and we will denote again by $X$ its set of vertices (while we will make use of the structure of $\mathcal{X}$ as a simplicial complex).

Recall that $\Gamma$ (hence, $\Gamma_{0}$ ) acts freely on $X$, so the bounded cohomology of $\Gamma_{0}$ may be isometrically computed via the complex

$$
C_{b}^{n}\left(\Gamma_{0} \curvearrowright X\right):=\left\{\varphi: X^{n+1} \rightarrow \mathbb{R},\|\varphi\|_{\infty}<\infty\right\}
$$

introduced in Section 1, For every Lipschitz map $f: \mathbb{Z} \rightarrow \mathbb{R}$ we are going to construct a 2-quasi-cocycle $\alpha_{f} \in \mathrm{QZ}_{\text {alt }}^{2}\left(\Gamma_{0} \curvearrowright X\right)^{\Gamma_{0}}$. The quasi-cocycle $\alpha_{f}$ should be understood as a discrete approximation of a primitive of a volume form on the infinite cyclic covering $M_{0}=\mathbb{H}^{3} / \Gamma_{0}$ of the cusped hyperbolic manifold $M=\mathbb{H}^{3} / \Gamma$ (here we are identifying $\Gamma$ with its realization as a non-uniform lattice in the isometry group of $\mathbb{H}^{3}$ ).

Recall that $M_{0}$ is diffeomorphic to $\Sigma \times \mathbb{R}$, where $\Sigma$ is a once-punctured torus. If $\sigma$ is any 2-simplex in $M_{0}$, then the evaluation on $\sigma$ of the primitive of a volume form on $M_{0}$ is equal to the volume of the prism spanned by $\sigma$ and by the projection of $\sigma$ on $\Sigma \times\{0\}$. Our construction in inspired by this remark, yet it is completely independent from the differential geometric situation just recalled. We define a projection $p: X \rightarrow \Gamma_{0}$ as follows: every element $X$ admits a unique expression as a pair $\left(g_{0} t^{k}, n\right)$ with $g_{0} \in \Gamma_{0}$, and we then set

$$
p: X \rightarrow \Gamma_{0}, \quad p\left(g_{0} t^{k}, n\right)=g_{0} .
$$

4.1. Heuristic. In this subsection we just discuss the geometric meaning of $p$, the reader may safely skip ahead if the point is clear already.

The projection $p: X \rightarrow \Gamma_{0}$ plays the role of the retraction of $M_{0}$ onto $\Sigma$. When considering $\Gamma$ as a non-uniform hyperbolic lattice, the action of $t$ on $\widetilde{M} \cong \widetilde{\Sigma} \times \mathbb{R}$ corresponds to the product of the lift of the pseudo-Anosov homeomorphism corresponding to $\psi$ (on $\widetilde{\Sigma}$ ) with the translation by 1 (on $\mathbb{R})$. Via the quasi-isometric identification between $X$ and $\widetilde{M}$, this action translates into the left action of $\Gamma$ on $X$. Observe now that the the group $\Gamma$ acts on $X$ also on the right as follows:

$$
(g, n) \cdot g^{\prime}=\left(g g^{\prime}, n\right) .
$$


This action is not by isometries, and it does not extend to a simplicial action over $X$ and neither over $\mathcal{X}$. However, from the equality $\operatorname{tgt}^{-1}=\psi(g), g \in \Gamma_{0}$, we deduce that the right action of $t$ on $X$ should correspond to the unitary translation on $\widetilde{\Sigma} \times \mathbb{R}$. Whence, the definition of $p$.

4.2. The combinatorial area form. In order to compute (signed) volumes, we need to introduce an orientation on triples in $X^{3}$. Let us fix a finite-area hyperbolization of $\Gamma_{0}$, i.e. a discrete faithful representation $\rho: \Gamma_{0} \rightarrow \operatorname{Isom}^{+}\left(\mathbb{H}^{2}\right)$ such that $\mathbb{H}^{2} / \rho\left(\Gamma_{0}\right)$ is isometric to a finite-volume oncepunctured torus, and denote by $\cdot$ the action of $\Gamma$ on $\partial \mathbb{H}^{2}$ induced by $\rho$. We identify $\partial \mathbb{H}^{2}$ with the topological boundary of the Poincaré disk, and we say that a triple of pairwise distinct points $\left(a_{0}, a_{1}, a_{2}\right)$ in $\partial \mathbb{H}^{2}$ is positive (resp. negative) if $\left(a_{0}, a_{1}, a_{2}\right)$ are anti-clockwise (resp. clockwise) oriented on $\partial \mathbb{H}^{2}$. Finally, if the points in the triple $\left(a_{0}, a_{1}, a_{2}\right) \in\left(\partial \mathbb{H}^{2}\right)^{3}$ are not pairwise distinct, we say that the triple is degenerate. The element $\rho([a, b])$ is parabolic, so it has a unique fixed point $\bar{q} \in \partial \mathbb{H}^{2}$. We then define a map $\varepsilon: \Gamma_{0}^{3} \rightarrow\{-1,0,1\}$ as follows:

$$
\varepsilon\left(g_{0}, g_{1}, g_{2}\right)=\left\{\begin{aligned}
1 & \text { if }\left(g_{0} \cdot \bar{q}, g_{1} \cdot \bar{q}, g_{2} \cdot \bar{q}\right) \text { is positive } \\
0 & \text { if }\left(g_{0} \cdot \bar{q}, g_{1} \cdot \bar{q}, g_{2} \cdot \bar{q}\right) \text { is degenerate } \\
-1 & \text { if }\left(g_{0} \cdot \bar{q}, g_{1} \cdot \bar{q}, g_{2} \cdot \bar{q}\right) \text { is negative. }
\end{aligned}\right.
$$

We extend $\varepsilon$ to a map defined on $X^{3}$ by setting:

$$
\varepsilon\left(x_{0}, x_{1}, x_{2}\right)=\varepsilon\left(p\left(x_{0}\right), p\left(x_{1}\right), p\left(x_{2}\right)\right) .
$$

The following result states that $\varepsilon$ is a $\Gamma$-invariant bounded cocycle:

Proposition 4.1. We have

$$
\varepsilon \in Z_{b}^{2}(\Gamma \curvearrowright X)^{\Gamma} .
$$

Moreover, if $x_{0}, x_{1}, x_{2}$ all lie in a 0 -horoball $\mathcal{H}$ of $\mathcal{X}$, then $\varepsilon\left(x_{0}, x_{1}, x_{2}\right)=0$.

Proof. The fact that $\varepsilon$ is a cocycle is easily checked. In order to prove that $\varepsilon$ is $\Gamma$-invariant it suffices to check that $g \cdot \varepsilon=\varepsilon$ for every $g \in \Gamma_{0}$, and $t \cdot \varepsilon=\varepsilon$.

Let us fix $g \in \Gamma_{0}$. It readily follows from the definition of $p$ that $p(g x)=$ $g p(x)$ for every $x \in X$. Therefore, for every triple $\left(x_{0}, x_{1}, x_{2}\right) \in X^{3}$ we have

$$
\left(p\left(g x_{0}\right) \cdot \bar{q}, p\left(g x_{1}\right) \cdot \bar{q}, p\left(g x_{2}\right) \cdot \bar{q}\right)=\left(g p\left(x_{0}\right) \cdot \bar{q}, g p\left(x_{1}\right) \cdot \bar{q}, g p\left(x_{2}\right) \cdot \bar{q}\right),
$$

and the conclusion easily follows from the fact that $\rho(g)$ acts on $\partial \mathbb{H}^{2}$ as an orientation-preserving homeomorphism.

In order to prove invariance with respect to $t$, first observe that the pseudo-Anosov homeomorphism $h: \Sigma \rightarrow \Sigma$ lifts to a quasi-isometry $\widetilde{h}: \mathbb{H}^{2} \rightarrow$ $\mathbb{H}^{2}$ such that

$$
\widetilde{h} \circ \rho(g)=\rho(\psi(g)) \circ \widetilde{h}
$$

for every $g \in \Gamma_{0}$. The quasi-isometry $\widetilde{h}$ continuously extends to $\partial \mathbb{H}^{2}$, and equation (2) also holds when considering the actions of $\widetilde{h}$ and of $\Gamma_{0}$ on $\partial \mathbb{H}^{2}$. 
In particular, if we set $g=[a, b]$ and we evaluate at $\bar{q}$ we obtain

$$
\widetilde{h}(\bar{q})=\widetilde{h}(g \cdot \bar{q})=\psi(g) \cdot(\widetilde{h}(\bar{q}))=g \cdot(\widetilde{h}(\bar{q})),
$$

so $\widetilde{h}(\bar{q})$ is fixed by $g$, and

$$
\widetilde{h}(\bar{q})=\bar{q} .
$$

Now, for every $x=\left(g_{0} t^{k}, n\right) \in X$ with $g_{0} \in \Gamma_{0}$, we have

$$
p(t x)=p\left(t\left(g_{0} t^{k}, n\right)\right)=p\left(\operatorname{tg}_{0} t^{k}, n\right)=p\left(\psi\left(g_{0}\right) t^{k+1}, n\right)=\psi\left(g_{0}\right)=\psi(p(x)),
$$

hence

$$
p(t x) \cdot \bar{q}=\psi(p(x)) \cdot \bar{q}=\psi(p(x)) \cdot \widetilde{h}(\bar{q})=\widetilde{h}(p(x) \cdot \bar{q}) .
$$

Let us now consider a triple $\left(x_{0}, x_{1}, x_{2}\right) \in X^{3}$. Observe that the trace of $\widetilde{h}$ on $\partial \mathbb{H}^{2}$ is an orientation-preserving homeomorphism. Therefore, the triple $\left(p\left(x_{0}\right) \cdot \bar{q}, p\left(x_{1}\right) \cdot \bar{q}, p\left(x_{2}\right) \cdot \bar{q}\right)$ is positive (resp. negative, degenerate) if and only

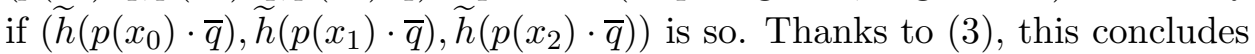
the proof that $\varepsilon$ is $t$-invariant, whence $\Gamma$-invariant.

Suppose now that $x_{0}, x_{1}, x_{2}$ all lie in the same horoball $\mathcal{H}$ of $X$. Then $p\left(x_{0}\right), p\left(x_{1}\right), p\left(x_{2}\right)$ all lie in the same left coset of $\langle[a, b]\rangle$ in $\Gamma_{0}$. Using again that $\bar{q}$ is fixed by $\rho([a, b])$, this implies that $p\left(x_{0}\right) \cdot \bar{q}=p\left(x_{1}\right) \cdot \bar{q}=p\left(x_{2}\right) \cdot \bar{q}$, so $\varepsilon\left(x_{0}, x_{1}, x_{2}\right)=0$.

4.3. The quasi-cocycle associated to a Lipschitz function. Let us now fix a Lipschitz function

$$
f: \mathbb{Z} \rightarrow \mathbb{R}
$$

We are going to define the quasi-cocycle $\alpha_{f}=\alpha(f) \in \mathrm{QZ}_{\text {alt }}^{2}(\Gamma \curvearrowright X)^{\Gamma_{0}}$ required in Theorem 5. The decomposition $\Gamma=\Gamma_{0} \rtimes_{\psi} \mathbb{Z}$ of $\Gamma$ as a semidirect product defines an epimorphism $\theta: \Gamma \rightarrow \mathbb{Z}$ given by $\theta(g)=k$, where $g=g_{0} t^{k}$ is the unique expression of $g$ such that $g_{0} \in \Gamma_{0}$. We extend $\theta$ to the whole of $X$ by setting $\theta(g, n)=\theta(g)$.

We first define the simplicial cochain $F_{f} \in C_{\Delta \text {,alt }}^{2}(\mathcal{X})$ such that, if $\sigma$ is a 2 -simplex in $\mathcal{X}$ with vertices $\left(x_{0}, x_{1}, x_{2}\right) \in X^{3}$, then

$$
F_{f}(\sigma)=\varepsilon\left(x_{0}, x_{1}, x_{2}\right) \frac{\sum_{i=0}^{2} f\left(\theta\left(x_{i}\right)\right)}{3} .
$$

Lemma 4.2. We have $F_{f} \in C_{\Delta, \text { alt }}^{2}(\mathcal{X})^{\Gamma_{0}}$. Moreover:

(1) $F_{f}(\tau)=0$ for every 2 -simplex $\tau$ contained in a horoball,

(2) $\left|F_{f}(\partial \sigma)\right| \leq R \cdot \operatorname{Lip}(f)$ for every 3 -simplex $\sigma$ of $\mathcal{X}$.

Proof. The fact that $F_{f}$ is alternating (resp. $\Gamma_{0}$-invariant) follows from the fact that $\varepsilon$ is (resp. that $\varepsilon$ and $\theta$ are). Moreover, if the 2-simplex $\tau=$ $\left(x_{0}, x_{1}, x_{2}\right)$ is contained in a horoball, then Proposition 4.1 implies $\varepsilon\left(x_{0}, x_{1}, x_{2}\right)=$ 0 , so $F_{f}(\tau)=0$.

Let now $\left(x_{0}, \ldots, x_{3}\right)$ be the vertices of a 3 -simplex $\sigma$. Recall that $\mathcal{X}=$ $\mathcal{R}_{\kappa}(X)$, where $\kappa=4 \delta+6$ and $\delta$ is a hyperbolicity constant for $X$. Since $x_{i}$ and $x_{j}$ are the vertices of a simplex in $\mathcal{X}$ we have $d\left(x_{i}, x_{j}\right) \leq \kappa$. If 
$\max D(\sigma) \geq \kappa+1$, then $\sigma$ is contained in a horoball $\mathcal{H}$, and hence $F_{f}(\partial \sigma)=$ 0 . In particular we can assume $\max D(\sigma) \leq \kappa$. By definition, if $m=$ $\sum_{j=0}^{3} f\left(x_{j}\right)$, then

$$
F_{f}\left(\partial_{i} \sigma\right)=\frac{\varepsilon\left(x_{0}, \ldots, \widehat{x_{i}}, \ldots, x_{3}\right)}{3}\left(m-f\left(\theta\left(x_{i}\right)\right)\right)
$$

so, using that $\sum_{i=0}^{3}(-1)^{i} \varepsilon\left(x_{0}, \ldots, \widehat{x_{i}}, \ldots x_{3}\right)=0$, we get:

$$
\begin{aligned}
F_{f}(\partial \sigma) & =\sum_{i=0}^{3}(-1)^{i}\left(\frac{\varepsilon\left(x_{0}, \ldots, \widehat{x_{i}}, \ldots, x_{3}\right)}{3}\left(m-f\left(\theta\left(x_{i}\right)\right)\right)\right) \\
& =-\sum_{i=0}^{3}(-1)^{i} \frac{\varepsilon\left(x_{0}, \ldots, \widehat{x_{i}}, \ldots, x_{3}\right)}{3} f\left(\theta\left(x_{i}\right)\right) .
\end{aligned}
$$

Using again that $\varepsilon$ is a cocycle with values in $\{-1,0,1\}$ we obtain that, up to a suitable permutation of $\left(x_{0}, x_{1}, x_{2}, x_{3}\right)$, either $F_{f}(\partial \sigma)=0$, or $F_{f}(\partial \sigma)=$ $f\left(\theta\left(x_{2}\right)\right)-f\left(\theta\left(x_{3}\right)\right)$, or $F_{f}(\partial \sigma)=f\left(\theta\left(x_{0}\right)\right)-f\left(\theta\left(x_{1}\right)\right)+f\left(\theta\left(x_{2}\right)\right)-f\left(\theta\left(x_{3}\right)\right)$. In any case, in order to conclude it is sufficient to show that $\mid f\left(\theta\left(x_{i}\right)\right)-$ $f\left(\theta\left(x_{j}\right)\right) \mid \leq R / 2$ for a universal constant $R$ for every fixed pair of indices $i, j \in\{0, \ldots, 3\}$.

First observe that, being a homomorphism, the restriction of $\theta$ to $\Gamma$ is $h$-Lipschitz for some $h>0$. Recall that $d$ denotes the distance on $X$, and denote by $d_{\Gamma}$ the distance on the Cayley graph of $\Gamma$ with respect to the fixed generating set $S$. If $x_{i}=\left(g_{i}, n_{i}\right), x_{j}=\left(g_{j}, n_{j}\right)$, with $n_{i}, n_{j} \leq \kappa$, we claim that $d_{\Gamma}\left(g_{i}, g_{j}\right) \leq \kappa 2^{2 \kappa}$ : indeed since $d\left(x_{1}, x_{2}\right) \leq \kappa$, any vertex in a geodesic in $X$ between $x_{1}$ and $x_{2}$ has depth at most $2 \kappa$, hence a geodesic in $X$ between $x_{1}$ and $x_{2}$ projects to a path in $\Gamma$ of length at most $\kappa 2^{2 \kappa}$. The conclusion follows if we set $R / 2=h \kappa 2^{2 \kappa}$.

We are now ready to define the quasi-cocycle $\alpha_{f}$. For every triple $\left(x_{0}, x_{1}, x_{2}\right) \in$ $X^{3}$ we set

$$
\alpha_{f}\left(x_{0}, x_{1}, x_{2}\right)=F_{f}\left(\varphi\left(x_{0}, x_{1}, x_{2}\right)\right),
$$

where $\varphi$ is the relative filling from Proposition 3.11 .

Proposition 4.3. We have

(1) $\alpha_{f} \in \mathrm{QZ}_{\text {alt }}^{2}\left(\Gamma_{0} \curvearrowright X\right)^{\Gamma_{0}}$,

(2) $\operatorname{def}\left(\alpha_{f}\right) \leq K \cdot \operatorname{Lip}(f)$ for a universal constant $K$.

Proof. The $\Gamma_{0}$-invariance of $\alpha_{f}$ follows from the $\Gamma_{0}$-invariance of $\varphi$ (which, indeed, is even $\Gamma$-invariant) and of $F_{f}$. Moreover, $\alpha_{f}$ is alternating, since both $F_{f}$ and $\varphi$ are. 
In order to bound the defect of $\alpha_{f}$, let us fix a quadruple $\left(x_{0}, \ldots, x_{3}\right) \in$ $X^{4}$, and estimate the value

$$
\begin{aligned}
\delta \alpha_{f}\left(x_{0}, x_{1}, x_{2}, x_{3}\right) & =\sum_{i=0}^{3}(-1)^{i} \alpha_{f}\left(x_{0}, \ldots, \widehat{x_{i}}, \ldots, x_{3}\right) \\
& =\sum_{i=0}^{3}(-1)^{i} F_{f}\left(\varphi\left(x_{0}, \ldots, \widehat{x_{i}}, \ldots, x_{3}\right)\right) \\
& =F_{f}\left(\sum_{i=0}^{3}(-1)^{i} \varphi\left(x_{0}, \ldots, \widehat{x_{i}}, \ldots, x_{3}\right)\right) .
\end{aligned}
$$

Proposition 3.11 ensures the existence of a relative filling of the chain $\sum_{i=0}^{3}(-1)^{i} \varphi\left(x_{0}, \ldots, \widehat{x_{i}}, \ldots, x_{3}\right)$ : we can choose a 3 -chain $B \in C_{3}^{\Delta}(\mathcal{X})_{\text {red }}$ with $\|B\|_{1} \leq T_{3}$ and such that the difference $\partial B-\sum_{i=0}^{3}(-1)^{i} \varphi\left(x_{0}, \ldots, \widehat{x_{i}}, \ldots, x_{3}\right)$ is a sum of simplices of $\mathcal{X}$ contained in a union of 0 -horoballs. We write $B=\sum_{j} \lambda_{j} \sigma_{j}$, with $\sum_{j}\left|\lambda_{j}\right| \leq T_{3}$. Since $F_{f}(\sigma)=0$ if $\sigma$ is a simplex contained in a 0 -horoball $\mathcal{H}$, we have:

$$
\begin{aligned}
\left|\delta \alpha_{f}\left(\left(x_{0}, x_{1}, x_{2}, x_{3}\right)\right)\right| & =\left|F_{f}(\partial B)-F_{f}\left(\partial B-\sum_{i=0}^{3}(-1)^{i} \varphi\left(x_{0}, \ldots, \widehat{x}_{i}, \ldots, x_{3}\right)\right)\right| \\
& =\left|F_{f}(\partial B)\right|=\left|\sum_{j} \lambda_{j} F_{f}\left(\partial \sigma_{j}\right)\right| \leq \sum_{j}\left|\lambda_{j}\right| \cdot\left|F_{f}\left(\partial \sigma_{j}\right)\right| \\
& \leq R \cdot \operatorname{Lip}(f) \cdot \sum_{j}\left|\lambda_{j}\right| \leq R \cdot T_{3} \cdot \operatorname{Lip}(f),
\end{aligned}
$$

where $R$ is the constant provided by Lemma4.2. The conclusion follows.

4.4. The 2-cycle $A_{m}$. Purpose of this section is to construct, for each $m \in \mathbb{N}$, a cycle $A_{m} \in C_{2}\left(\Gamma_{0} \curvearrowright X\right)_{\text {red, } \Gamma_{0}}$ on which we will evaluate our cocycles $\alpha_{f}$.

In what follows we will omit, for ease of notation, to distinguish a chain in $C_{n}\left(\Gamma_{0} \curvearrowright X, \mathbb{R}\right)_{\Gamma_{0}}$ from its reduced image in $C_{n}\left(\Gamma_{0} \curvearrowright X, \mathbb{R}\right)_{\text {red, } \Gamma_{0}}$. For example, we will simply write $(x, y)=-(y, x)$ for every $(x, y) \in X^{2}$. We will construct the cycle $A_{m}$ as a union of different combinatorial analogues of geometric pieces.

Let $e \in \Gamma$ denote the identity element. The combinatorial analogue of a relative fundamental class for $\Gamma_{0} \subseteq X$ is $c:=((e, 0),(b, 0),(b a, 0))+((e, 0),(b a, 0),(a b, 0))+((e, 0),(a b, 0),(a, 0))$.

An easy computation in $C_{2}\left(\Gamma_{0} \curvearrowright X\right)_{\mathrm{red}, \Gamma_{0}}$ gives

$$
\partial c=((e, 0),([a, b], 0))=((b a, 0),(a b, 0)) .
$$




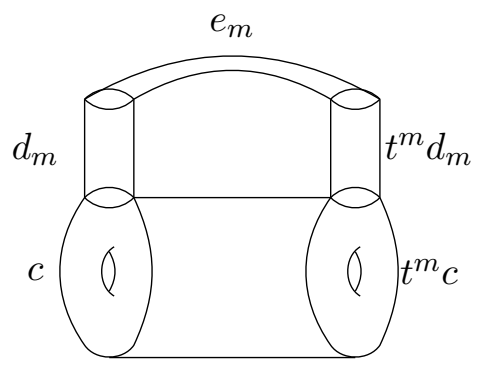

Figure 1. The 2-cycle $A_{m}$

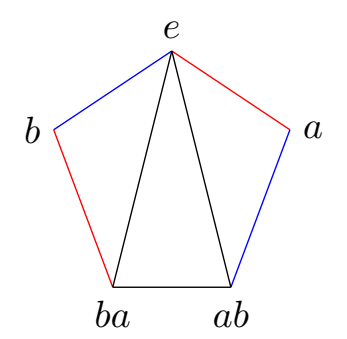

Figure 2. The relative fundamental class $c$

The second building block of our cycle $A_{m}$ is the combinatorial counterpart of a small annulus going deep enough into the horoball. We will need this to be able to join the boundaries of two combinatorial fundamental classes with two simplices. We will prove that, for each $K \in \mathbb{N}$, the 1-cycle $\partial c$ is homologous to the 1-cycle $a_{K}:=\frac{1}{2^{K}}\left((e, K),\left([a, b]^{2^{K}}, K\right)\right)$.

We choose $K_{m}:=\left\lfloor\log _{2} m\right\rfloor+1$ big enough, so that $d\left(\left(e, K_{m}\right),\left(t^{m}, K_{m}\right)\right)=$ 1 , and consider the chain

$$
\begin{aligned}
d_{m}:=\sum_{i=0}^{K_{m}-1} \frac{1}{2^{i+1}} & \left(\left((e, i),(e, i+1),\left([a, b]^{2^{i}}, i\right)\right)+\right. \\
& +\left(\left([a, b]^{2^{i}}, i\right),(e, i+1),\left([a, b]^{2^{i+1}}, i+1\right)\right)+ \\
& \left.+\left(\left([a, b]^{2^{i}}, i\right),\left([a, b]^{2^{i+1}}, i+1\right),\left([a, b]^{2^{i+1}}, i\right)\right)\right) .
\end{aligned}
$$

In $C_{2}(X, \mathbb{R})_{\text {red, } \Gamma_{0}}$, we have

$$
\partial d_{m}=-\partial c+a_{K_{m}}
$$

which, in particular, proves that $\partial c$ and $a_{K_{m}}$ are homologous. 


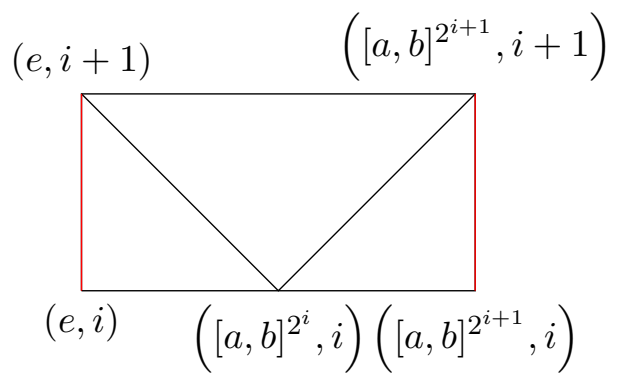

Figure 3. A portion of the annulus $d_{m}$

Our third and last building block is an annulus supported deep in the horoball with boundary $a_{K_{m}}-t^{m} a_{K_{m}}$. We will call it $e_{m}$ :

$$
\begin{aligned}
e_{m}:=\frac{1}{2^{K_{m}}} & \left(\left(\left(e, K_{m}\right),\left(t^{m}[a, b]^{2^{K_{m}}}, K_{m}\right),\left(t^{m}, K_{m}\right)\right)\right. \\
& \left.+\left((e, K),\left([a, b]^{2^{K_{m}}}, K_{m}\right),\left(t^{m}[a, b]^{2^{K_{m}}}, K_{m}\right)\right)\right) .
\end{aligned}
$$

In order to verify that $\partial e_{m}=a_{K_{m}}-t^{m} a_{K_{m}}$, we use that the pseudo-Anosov $\psi$ fixes the commutator $[a, b]$ and hence in particular $t^{m}[a, b]^{2^{K_{m}}}=[a, b]^{2^{K_{m}}} t^{m}$.

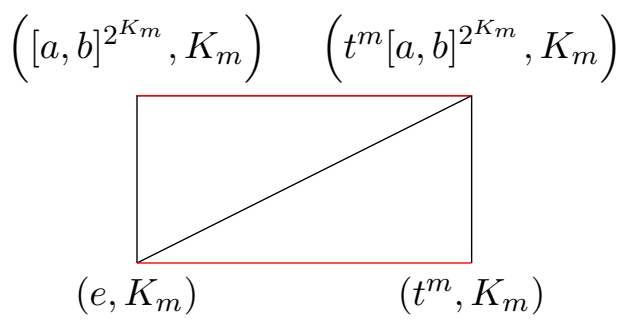

Figure 4. The annulus $e_{m}$

We can now define

$$
A_{m}:=t^{m} \cdot\left(c+d_{m}\right)-\left(c+d_{m}\right)+e_{m} .
$$

Lemma 4.4. Let $m \geq 0$. Then

(1) $\left\|A_{m}\right\|_{1} \leq 9$;

(2) the chain $A_{m}$ is a boundary in $C_{2}\left(\Gamma_{0} \curvearrowright X\right)_{r e d, \Gamma_{0}}$.

Proof. (1): We have

$$
\begin{aligned}
\left\|A_{m}\right\|_{1} & \leq 2\|c\|_{1}+2\left\|d_{m}\right\|_{1}+\left\|e_{m}\right\|_{1} \\
& \leq 6+3 \sum_{i=0}^{K_{m}-1} 2^{-i-1}+2 \cdot 2^{-K_{m}} \leq 6+3 \sum_{i=0}^{\infty} 2^{-i-1}=9 .
\end{aligned}
$$


THE ZERO NORM SUBSPACE FOR ACYLINDRICALLY HYPERBOLIC GROUPS 36

(2): We already verified that $\partial A_{m}=0$. Observe that each simplex involved in the definition of $A_{m}$ exists in the Rips complex $\mathcal{X}$ and hence $A_{m}$ also defines a cycle as a simplicial chain in $C_{2}^{\Delta}(\mathcal{X})_{\text {red, } \Gamma_{0}}=C_{2}^{\Delta}\left(\mathcal{X} / \Gamma_{0}\right)_{\text {red }}$. Since the Rips complex $\mathcal{X}$ is contractible, the simplicial homology of $\mathcal{X} / \Gamma_{0}$ is canonically isomorphic to the homology of $\Gamma_{0} \cong F_{2}$, which of course vanishes in degree 2. Therefore, there exist a simplicial 3-chain $B_{m} \in C_{3}^{\Delta}(\mathcal{X})_{\text {red, } \Gamma_{0}}$ with $\partial B_{m}=A_{m}$. The chain $B_{m}$ also defines an element of $C_{3}\left(\Gamma_{0} \curvearrowright X\right)_{\mathrm{red}, \Gamma_{0}}$ with $\partial B_{m}=A_{m}$.

4.5. Proof of Theorem 5. We now turn to the proof of Theorem 5 that we recall for the reader's convenience:

Theorem 4.5. Let $\mathcal{L}(\mathbb{Z}, \mathbb{R})$ be the space of Lipschitz real functions on $\mathbb{Z}$. There exist a constant $C>0$ and a linear map

$$
\alpha: \mathcal{L}(\mathbb{Z}, \mathbb{R}) \rightarrow \mathrm{QZ}_{\text {alt }}^{2}\left(\Gamma_{0} \curvearrowright X\right)^{\Gamma_{0}}
$$

such that the following conditions hold:

(1) $\operatorname{def}(\alpha(f))=\| \delta \alpha(f)) \|_{\infty} \leq C \cdot \operatorname{Lip}(f)$ for every $f \in \mathcal{L}(\mathbb{Z}, \mathbb{R})$;

(2) $[\delta \alpha(f)]=0$ in $H_{b}^{3}\left(\Gamma_{0} \curvearrowright X\right) \cong H_{b}^{3}\left(\Gamma_{0}\right)$ if and only if $f$ is bounded.

Of course the map

$$
\begin{array}{ccc}
\alpha: \mathcal{L}(\mathbb{Z}, \mathbb{R}) & \rightarrow & \mathrm{QZ}_{\text {alt }}^{2}\left(\Gamma_{0} \curvearrowright X\right)^{\Gamma_{0}} \\
f & \mapsto & \alpha_{f}
\end{array}
$$

defined in Section 4.3 is linear, and we proved (1) in Proposition 4.3, The last missing step in the proof of Theorem 5 is:

Proposition 4.6. $\delta \alpha_{f}$ represents 0 in $H_{b}^{3}\left(\Gamma_{0} \curvearrowright X\right)$ if and only if the Lipschitz function $f$ is bounded.

In order to prove Proposition 4.6 we need to compute the value of $\alpha_{f}$ on $A_{m}$. We begin with a preliminary lemma:

Lemma 4.7. Let $a, b$ be the generators of $\Gamma_{0}$. We have $\varepsilon(e, a b, a)=\varepsilon(e, b, b a)=$ \pm 1 and $\varepsilon(e, b a, a b)=0$.

Proof. Recall that, in order to define $\varepsilon$, we chose a finite area hyperbolization $\rho$ of $\Gamma_{0}$, and we denoted by $\bar{q} \in \partial \mathbb{H}^{2}$ the unique fixed point of $\rho([a, b])=$ $\rho\left(a^{-1} b^{-1} a b\right)$. By definition, $\pi \varepsilon\left(g_{0}, g_{1}, g_{2}\right)$ is the area of the ideal triangle in $\mathbb{H}^{2}$ with vertices $\left(g_{0} \cdot \bar{q}, g_{1} \cdot \bar{q}, g_{2} \cdot \bar{q}\right)$.

Since $b a \cdot \bar{q}=b a \cdot([a, b] \cdot \bar{q})=a b \cdot \bar{q}$, the ideal triangle with vertices $(\bar{q}, b a \cdot \bar{q}, a b \cdot \bar{q})$ is degenerate, and hence $\varepsilon(e, b a, a b)=0$. Moreover the union of the two triangles $(\bar{q}, a b \cdot \bar{q}, a \cdot \bar{q})$ and $(\bar{q}, b \cdot \bar{q}, b a \cdot \bar{q})$ is a fundamental domain for the $\Gamma_{0}$ action on $\mathbb{H}^{2}$, and hence $\varepsilon(e, a b, a)=\varepsilon(e, b, b a)$ are non-zero. The common sign depends on the choice of the generators $a, b$.

The next lemma shows that the cycle $A_{m}$ encloses a volume proportional to $m$ :

Lemma 4.8. $\left|\alpha_{f}\left(A_{m}\right)\right|=2|f(m)-f(0)|$. 
Proof. We evaluate $\alpha_{f}\left(A_{m}\right)$ term by term. Observe that every simplex in the support of $A_{m}$ has vertices at distance at most 2 , and there exists a horoball $\mathcal{H}$ such that

$$
\operatorname{Supp}\left(d_{m}+e_{m}+t^{m} d_{m}\right) \subset \mathcal{H} .
$$

It then follows from Proposition 3.11 (2) that, for each simplex $\sigma$ in the support of $t^{m} d_{m}+e_{m}-d_{m}$, we have $\varphi(\sigma) \subset \mathcal{H}$ and hence

$$
\alpha_{f}\left(t^{m} d_{m}+e_{m}-d_{m}\right)=F_{f}\left(\varphi\left(t^{m} d_{m}+e_{m}-d_{m}\right)\right)=0
$$

by Lemma 4.2 (1). Therefore $\alpha_{f}\left(A_{m}\right)=\alpha_{f}\left(t^{m} c-c\right)$.

We know from Lemma 4.7 that $\varepsilon(e, a b, a)=\varepsilon(e, b, b a)= \pm 1$ and $\varepsilon(e, b a, a b)=$ 0 . Since $\varepsilon$ is $\Gamma$-invariant (Proposition 4.1), and hence in particular $t$-invariant, we also deduce $\varepsilon\left(t^{m}, t^{m} a b, t^{m} a\right)=\varepsilon(e, a b, a)$. Moreover, since all simplices involved in the definition of $c$ and $t^{m} c$ have vertices at distance at most 1 , Proposition 3.11 (1) implies that $\varphi$ is the identity on each of them. We now have $\theta(v)=m$ for every vertex $v$ in $\operatorname{Supp}\left(t^{m} c\right)$, and $\theta(v)=0$ for every vertex $v$ in $\operatorname{Supp}(c)$, so $\alpha_{f}\left(t^{m} c\right)-\alpha_{f}(c)=2 \varepsilon(e, a b, a)(f(m)-f(0))$, and this concludes the proof.

Proof of Proposition 4.6. Suppose that $f$ is bounded. For every $\left(x_{0}, x_{1}, x_{2}\right) \in$ $X^{3}$, we write

$$
\varphi\left(x_{0}, x_{1}, x_{2}\right)=\sum_{\sigma} \lambda_{\sigma} \sigma
$$

with $\sum_{\sigma}\left|\lambda_{\sigma}\right| \leq T_{2}$ (see Proposition 3.11 (3)). Then

$$
\begin{aligned}
\left|\alpha_{f}\left(x_{0}, x_{1}, x_{2}\right)\right| & =\left|F_{f}\left(\varphi\left(x_{0}, x_{1}, x_{2}\right)\right)\right| \\
& \leq \frac{1}{3} \sum_{\sigma}\left|\lambda_{\sigma}\right| \sum_{i=0}^{2}\left|f\left(\theta\left(\sigma^{(i)}\right)\right)\right| \\
& \leq T_{2}\|f\|_{\infty} .
\end{aligned}
$$

This shows that $\delta \alpha_{f}$ is the coboundary of a bounded 2-cochain and hence represents 0 in $H_{b}^{3}\left(\Gamma_{0} \curvearrowright X\right)$.

Vice versa, suppose that $f$ is a Lipschitz function such that $\delta \alpha_{f}=\delta \beta$ for some bounded $\Gamma_{0}$-invariant cochain $\beta \in C_{b}^{2}\left(\Gamma_{0} \curvearrowright X\right)^{\Gamma_{0}}$. By Lemma 4.4. there exists a 3-chain $B_{m} \in C_{3}(X, \mathbb{R})_{\text {red, } \Gamma_{0}}$ with $\partial B_{m}=A_{m}$. Recall from Lemma 4.4 that $\left\|A_{m}\right\|_{1} \leq 9$, so

$$
\begin{aligned}
\left|\alpha_{f}\left(A_{m}\right)\right| & =\left|\alpha_{f}\left(\partial B_{m}\right)\right|=\left|\left(\delta \alpha_{f}\right)\left(B_{m}\right)\right|=\left|(\delta \beta)\left(B_{m}\right)\right| \\
& =\left|\beta\left(\partial B_{m}\right)\right|=\left|\beta\left(A_{m}\right)\right| \leq\|\beta\|_{\infty} \cdot\left\|A_{m}\right\| \leq 9\|\beta\|_{\infty} .
\end{aligned}
$$

Therefore, $\left|\alpha_{f}\left(A_{m}\right)\right|$ is uniformly bounded. By Lemma 4.8, this implies that $|f(m)| \leq\left|\frac{1}{2} \alpha_{f}\left(A_{m}\right)\right|+|f(0)|$ is also uniformly bounded, i.e. that $f$ is bounded, as desired. 
THE ZERO NORM SUBSPACE FOR ACYLINDRICALLY HYPERBOLIC GROUPS 38

4.6. Proof of Theorem 2. In order to conclude the proof of Theorem 2 we are now left to construct an uncountable set of linearly independent elements in $N_{0}^{3}\left(F_{2}\right) \subseteq H_{b}^{3}\left(F_{2}\right)$. For every $f \in \mathcal{L}(\mathbb{Z}, \mathbb{R})$ we set

$$
f_{n}(x):= \begin{cases}f(x)-f(-n) & \text { if } x \leq-n \\ 0 & \text { if }-n \leq x \leq n \\ f(x)-f(n) & \text { if } x \geq n\end{cases}
$$

and

$$
\mathcal{L}^{0}(\mathbb{Z}, \mathbb{R})=\left\{f \in \mathcal{L}(\mathbb{Z}, \mathbb{R}) \mid \lim _{n \rightarrow \infty} \operatorname{Lip}\left(f_{n}\right)=0\right\} .
$$

We choose the basepoint $x=(e, 0) \in X$ and we set

$$
\eta: \mathcal{L}(\mathbb{Z}, \mathbb{R}) \rightarrow H_{b}^{3}\left(\Gamma_{0}\right), \quad \eta(f)=w_{x}^{3}\left(\left[\delta \alpha_{f}\right]\right),
$$

where $w_{x}^{3}: H_{b}^{3}\left(\Gamma_{0} \curvearrowright X\right) \rightarrow H_{b}^{3}\left(\Gamma_{0}\right)$ is the map described in Lemma 1.1.

Recall from Lemma 1.1 that the complex $C_{b, \text { alt }}^{*}\left(\Gamma_{0} \curvearrowright X\right)^{\Gamma_{0}}$ isometrically computes the bounded cohomology of $\Gamma_{0}$, so by Proposition 4.6 the map $\eta$ induces an isomorphism between $\mathcal{L}^{0}(\mathbb{Z}, \mathbb{R}) /\left(\mathcal{L}^{0}(\mathbb{Z}, \mathbb{R}) \cap \ell^{\infty}(\mathbb{Z})\right)$ and $\eta\left(\mathcal{L}^{0}(\mathbb{Z}, \mathbb{R})\right) \subseteq H_{b}^{3}\left(\Gamma_{0}\right)$. It is immediate to realize that the dimension of the real vector space $\mathcal{L}^{0}(\mathbb{Z}, \mathbb{R}) /\left(\mathcal{L}^{0}(\mathbb{Z}, \mathbb{R}) \cap \ell^{\infty}(\mathbb{Z})\right)$ is equal to the cardinality of the continuum: for example, the classes of the maps $n \mapsto n^{\alpha}, \alpha \in(0,1)$, define linear independent elements in $\mathcal{L}^{0}(\mathbb{Z}, \mathbb{R}) /\left(\mathcal{L}^{0}(\mathbb{Z}, \mathbb{R}) \cap \ell^{\infty}(\mathbb{Z})\right)$. Therefore, Theorem 2 is now reduced to the following:

Proposition 4.9. For every function $f \in \mathcal{L}^{0}(\mathbb{Z}, \mathbb{R})$ we have $\eta(f) \in N_{0}^{3}\left(\Gamma_{0}\right)$.

Proof. For every $n \in \mathbb{N}$ we have $\left\|f-f_{n}\right\|_{\infty}=\max \left\{|f|_{[-n, n]} \mid\right\}<\infty$, hence by Proposition 4.6 we have $\eta\left(f_{n}\right)=\eta(f)$ for every $n \in \mathbb{N}$. Therefore, for every $n \in \mathbb{N}$ the cochain $w_{x}^{2}\left(\alpha_{f_{n}}\right) \in C^{2}\left(\Gamma_{0}\right)$ is a primitive of $\eta(f)$.

Let us fix the exhaustion $\left(S_{i}\right)_{i \in \mathbb{N}}$ of $\Gamma_{0}$ given by

$$
S_{i}=\left\{\gamma \in \Gamma_{0} \mid d_{\Gamma_{0}}(\gamma, e) \leq i\right\} .
$$

For every $i$, we can choose $n_{i}$ big enough so that $\left.w_{x}^{2}\left(\alpha_{f_{n_{i}}}\right)\right|_{S_{i}^{3}}=0$ : indeed the set $S_{i}$ is finite, and for each triple $\left(s_{0}, s_{1}, s_{2}\right) \in S_{i}^{3}$, the simplicial 2chain $\varphi\left(\left(s_{0}, 0\right),\left(s_{1}, 0\right),\left(s_{2}, 0\right)\right)$ involves only a finite number of simplices. In particular we can find $n_{i}$ such that $\left|\theta\left(\operatorname{Supp} \varphi\left(\left(s_{0}, 0\right),\left(s_{1}, 0\right),\left(s_{2}, 0\right)\right)\right)\right| \leq n_{i}$ for every $\left(s_{0}, s_{1}, s_{2}\right) \in S_{i}^{3}$, and for such $n_{i}$ we have $\left.w_{x}^{2}\left(\alpha_{f_{n_{i}}}\right)\right|_{S_{i}^{3}}=0$. Clearly we can also suppose that the sequence $\left\{n_{i}\right\}_{i \in \mathbb{N}}$ is monotonically diverging to $\infty$.

Recall now that Proposition 4.3 ensures that $\left\|\delta \alpha_{f_{n_{i}}}\right\|_{\infty} \leq K \operatorname{Lip}\left(f_{n_{i}}\right)$, so since $f \in \mathcal{L}^{0}(\mathbb{Z}, \mathbb{R})$ we have $\lim _{i \rightarrow \infty}\left\|\delta \alpha_{f_{n_{i}}}\right\|_{\infty}=0$. Therefore, since $w_{x}^{n}$ is norm non-increasing, by Lemma 2.6 we finally get

$$
\|\eta(f)\|_{\infty, 0} \leq \liminf _{i \rightarrow \infty}\left\|\delta w_{x}^{2}\left(\alpha_{f_{n_{i}}}\right)\right\|_{\infty} \leq \liminf _{i \rightarrow \infty}\left\|\delta \alpha_{f_{n_{i}}}\right\|_{\infty}=0 .
$$




\section{APPENDIX: VOLUMES OF MAPPING TORI}

We use the techniques introduced in the paper to give a cohomological proof of (some particular cases) of an inequality due to Brock Bro03b, Theorem 1.1].

Let $\Sigma_{g}$ be the closed oriented surface of genus $g, g \geq 2$. If $\psi: \Sigma_{g} \rightarrow \Sigma_{g}$ is a pseudo-Anosov homeomorphism, we denote by $M_{\psi}$ the mapping torus

$$
M_{\psi}:=\Sigma \times[0,1] /(x, 0) \equiv(\psi(x), 1) .
$$

Recall that a pseudo-Anosov homeomorphism $\psi: \Sigma_{g} \rightarrow \Sigma_{g}$ is $\epsilon$-cobounded if the image of its Teichmüller axis in the moduli space $\mathcal{M}_{g}$ stays in the $\epsilon$-thick part $\mathcal{M}_{g}^{\epsilon}[$ FM02, Section 2.1]. We denote by $\tau(\psi)$ the translation length of $\psi$ on the Teichmüller space endowed with the Teichmüller metric, which is well known to be equal to its maximal dilatation $\lambda$ Ber78. For $\psi \epsilon$-cobounded, it is well known that the Teichmüller translation length is also uniformly bi-Lipschitz equivalent to the Weil-Petersson translation length. This can be seen as follows. Distances in both the Teichmüller and the Weil-Petersson metric can be computed, up to bounded multiplicative and additive error, in terms of the so-called subsurface projections to curve complexes of subsurfaces; this is known as the distance formula, see [MM00, Theorem 6.12] Bro03a, Theorem 4.4] for the Weil-Petersson case and [Raf07, Theorem 1.1] for the Teichmüller case. The difference between the distance formulas is that annular subsurfaces do not contribute in the Weil-Petersson case, while they do in the Teichmüller case. As observed in, e.g., [KL07, Theorem 3.1], it follows from Raf05] that in the $\epsilon$-cobounded case all subsurface projections to curve complexes of proper subsurfaces are bounded, so that both in the Teichmüller and in the Weil-Petersson case the distance formula only has one non-zero term, the one corresponding to the whole surface, easily implying the desired relation between translation distances.

The purpose of the appendix is to give a different proof of the lower bound of the volume of $M_{\psi}$ in terms of the dilation of $\psi$, when $\psi$ is an $\epsilon$-cobounded pseudo-Anosov.

Theorem 5.1. There exists a constant $C>0$ depending only on $\epsilon$ and $g$ such that, for any $\epsilon$-cobounded pseudo-Anosov $\psi: \Sigma_{g} \rightarrow \Sigma_{g}$, we have

$$
\operatorname{vol}\left(M_{\psi}\right) \geq C \tau(\psi) \text {. }
$$

Remark 5.2. Brock proves [Bro03b, Theorem 1.1] that there is a constant $K$ depending only on the genus of the surface such that

$$
\frac{1}{K}\|\psi\|_{W P} \leq \operatorname{vol}\left(M_{\psi}\right) \leq K\|\psi\|_{W P}
$$

for every pseudo-Anosov homeomorphism $\psi: \Sigma_{g} \rightarrow \Sigma_{g}$, where $\|\cdot\|_{W P}$ denotes the translation length of $\psi$ with respect to the Weil-Petersson metric. The lower bound is deduced from the fact that in $M_{\psi}$ there are at least 
$\|\psi\|_{W P}$ short curves with disjoint Margulis tubes, each of which gives a definite contribution to the volume.

Upper bounds on the volume in terms of different translation lengths and with an explicit dependence on the genus are known: for any pseudo-Anosov $\psi: \Sigma \rightarrow \Sigma$, Kojima and McShane [KM14, Theorem 2 and Proposition 12] prove the inequality

$$
3 \pi|\chi(\Sigma)| \tau(\psi) \geq \operatorname{vol}\left(M_{\psi}\right)
$$

while Brock and Bromberg [BB16] prove that

$$
\sqrt{3 \pi / 2(2 g-2+n)}\|\psi\|_{W P} \geq \operatorname{vol}\left(M_{\psi}\right) .
$$

Proof summary. Denote by $\Gamma$ the fundamental group of $M_{\psi}$, and set $\Gamma_{0}=\pi_{1}\left(\Sigma_{g}\right)$ so that $\Gamma=\Gamma_{0} *_{*}$, where $\psi_{*}$ denotes the automorphism of $\Gamma_{0}$ induced by $\psi$. The strategy of our proof of Theorem 5.1 is based on the ideas developed in the main paper: we construct an explicit combinatorial cocycle representing some multiple of the volume form of the three manifold $M_{\psi}$ and we compute its value on a suitable fundamental class.

In order to define our cocycle, we will first construct, as in Section 4, a graph $X$ which is a discrete approximation of $\widetilde{M_{\psi}}$. As in the case of the graph considered in Section 4, $X$ admits a $\Gamma$-action, a $\Gamma_{0}$-equivariant projection $p: X \rightarrow \Gamma_{0}$, a $\Gamma$-equivariant, 1-Lipschitz projection $\theta: X \rightarrow \mathbb{R}$. Furthermore $X$ is uniformly $\delta$ hyperbolic and has uniformly bounded degree. Therefore, as a consequence of Mineyev's Theorem, a suitable Rips complex $\mathcal{X}$ over it admits a homological filling $\varphi: X^{3} \rightarrow C_{2}^{\Delta}(\mathcal{X})$ with uniformly bounded norm, and uniformly bounded filling (Lemma [5.5).

Using the same ideas as in Section 3 we use $\varphi$ to construct a combinatorial primitive of the volume form: a $\Gamma$-invariant quasi-cocycle $\alpha \in \mathrm{QZ}^{2}(\Gamma \curvearrowright X)$. In Section 5.3 we will use $\alpha$ to give a lower bound on the simplicial volume of $M_{\psi}$ and therefore on its hyperbolic volume.

5.1. The graph $X$, a combinatorial approximation of $M_{\psi}$. We assume (up to raising $\psi$ to a suitable power) that $\tau(\psi)$ is at least one. Let $\lambda$ be the sub-multiple of $\tau(\psi)$ in the interval $(0.5,1]$, set $k=\tau(\psi) / \lambda$.

We denote by $l$ the Teichmüller axis of $\psi$ and choose a basepoint 0 on $l \cong \mathbb{R}$. The group $\Gamma$ acts on the canonical $\mathbb{H}^{2}$-bundle over $l$ FM02, page 107] and in particular, for each $s$, the subgroup $\Gamma_{0}$ acts by isometries on the fiber at time $s$, that we denote by $\mathbb{H}_{s}^{2}$. We also choose a basepoint $b_{0}$ on $\mathbb{H}_{0}^{2}$. The unique isometric lift of $l$ through $b_{0}$ allows us to choose coherent basepoints $b_{s}$ for each fiber.

Lemma 5.3. There is a constant $C$, depending on $g$ and $\epsilon$ only, such that for any $s \in \mathbb{R}$ we have

$$
\operatorname{diam}\left(\mathbb{H}_{s}^{2} / \Gamma_{0}\right) \leq C .
$$

Proof. The homomorphism $\psi$ is $\epsilon$-cobounded, so every geodesic loop in $\Sigma_{s}=$ $\mathbb{H}_{s}^{2} / \Gamma_{0}$ has length at least $\epsilon$. This readily implies that the $\epsilon / 4$-neighborhood 
of any length-minimizing geodesic of $\Sigma_{s}$ is isometric to the $\epsilon / 4$-neighborhood of a geodesic of length $L$ in $\mathbb{H}^{2}$.

Since the area of the $\epsilon$-neighborhood of a geodesic of length $L$ grows linearly with $L$, and the area of $\Sigma_{s}$ is equal to $2 \pi \chi(\Sigma)$, this provides the desired upper bound on the lengths of minimizing geodesics in $\Sigma_{s}$, i.e. on the diameter of $\mathbb{H}_{s}^{2} / \Gamma_{0}$.

For each $n \in \mathbb{Z}$ denote by $X_{n}$ the graph whose vertex set is $\Gamma_{0} \times\{n\}$ and with the property that two vertices $(g, n),(h, n)$ are joined by an edge if and only if $d\left(g b_{\lambda n}, h b_{\lambda n}\right) \leq 6 C$. By Milnor-Svarc Lemma $X_{n}$ is $(6 C, 1)$ quasi-isometric to $\mathbb{H}_{\lambda n}^{2}$ (see, for example, the proof in BH99b, Proposition I.8.19]). Moreover $X_{n}$ has valency bounded above by $D(\epsilon, C)$. The graph $X$ is the union of the $X_{n}$ with horizontal edges of type $((g, n),(g, n+1))$.

Observe that there are a natural $\Gamma$-action on $X$, a natural projection $p: X^{(0)}=\Gamma_{0} \times \mathbb{Z} \rightarrow \Gamma_{0}$ and a natural 1-Lipschitz map $\theta: X^{(0)} \rightarrow \mathbb{R}$ defined by $\theta(g, n)=n \lambda$.

The graph $X$ is uniformly quasi-isometric to the canonical $\mathbb{H}^{2}$-bundle over $l$. Farb-Mosher [FM02, page 145] use Bestvina-Feighn's combination theorem to show:

Proposition 5.4. There exists $\delta=\delta(g, \epsilon)$ such that $X$ is $\delta$-hyperbolic.

Denote by $\mathcal{X}$ the Rips complex over $X$ with constant $\kappa \geq 4 \delta+6$. Since the graph $X$ is hyperbolic and has bounded valency, it admits a homological bicombing with a good filling $\varphi$ :

Lemma 5.5. There exist a constant $T_{3} \in \mathbb{R}$ depending on $\epsilon$ and $g$ only, and a $\Gamma$-equivariant map $\varphi: X^{3} \rightarrow C_{2}^{\Delta}(\mathcal{X})_{\text {red }}$ such that

(1) $\varphi\left(x_{0}, x_{1}, x_{2}\right)=\left[x_{0}, x_{1}, x_{2}\right]$ if $d\left(x_{i}, x_{i}\right) \leq \kappa$;

(2) for any 4-tuple $\left(x_{0}, x_{1}, x_{2}, x_{3}\right)$ of vertices of $X$ there exists $B \in$ $C_{3}^{\Delta}(\mathcal{X})$ with $\varphi\left(\partial\left(x_{0}, x_{1}, x_{2}, x_{3}\right)\right)=\partial B$ and $\|B\|_{1} \leq T_{3}$.

Proof. Since $X$ is $\delta$-hyperbolic and has uniformly bounded degree, Mineyev's construction gives a $\Gamma$-equivariant, anti-symmetric homological bicombing $\varphi_{1}: X^{2} \rightarrow C_{1}^{\Delta}(\mathcal{X})$ with the property that for each triple $\left(x_{0}, x_{1}, x_{2}\right)$, $\left\|\varphi_{1}\left(\partial\left(x_{0}, x_{1}, x_{2}\right)\right)\right\|_{1} \leq T_{1}(g, \epsilon)$ [Min01, Theorem 10] (cfr. also GM08, Theorem 6.2] where it is observed that the constant $T_{1}$ in Mineyev's construction only depends on the valency of the 1-skeleton of $X$ and its hyperbolicity constant). By [Min01, Proposition 12] there exists a filling $\varphi:=\varphi_{2}: X^{3} \rightarrow$ $C_{2}^{\Delta}(\mathcal{X})_{\text {red }}$ so that $\partial \varphi\left(x_{0}, x_{1}, x_{2}\right)=\varphi_{1}\left(x_{0}, x_{1}\right)+\varphi_{1}\left(x_{1}, x_{2}\right)+\varphi_{1}\left(x_{2}, x_{0}\right)$. Again by [Min01, property (2) holds with a constant $T_{3}$ depending only on the hyperbolicity constant $\delta$ and the valency. We are free to modify $\varphi$ on small simplices to ensure (1), because the norm of $\varphi_{1}\left(x_{0}, x_{1}\right)$ is bounded by a function of the distance of $x_{0}, x_{1}$ (this is part of Mineyev's definition of quasi-geodesic bicombing). 
THE ZERO NORM SUBSPACE FOR ACYLINDRICALLY HYPERBOLIC GROUPS 42

5.2. The primitive of the volume form $\alpha$. Just as we did in Section 4, in order to define a primitive $\alpha$ of a combinatorial volume form, we need to define a suitable sign $\epsilon$ for every triple of vertices of $X$.

Fix a hyperbolization $\rho: \Gamma_{0} \rightarrow \operatorname{Isom}\left(\mathbb{H}^{2}\right)$, and a lift $\widetilde{h}: \mathbb{H}^{2} \rightarrow \mathbb{H}^{2}$ of the pseudo-Anosov homeomorphism $\psi$ with a fixed point $\bar{q}$ in $\partial \mathbb{H}^{2}$. (Such a lift exists: consider a singular point $x \in \mathbb{H}^{2}$ for the lift of the singular foliation preserved by $\psi$. If we choose the lift $\widetilde{h}$ with the property that $\widetilde{h}(x)=x$, we get that the endpoints at infinity of the singular leaves through $x$ are fixed by $\widetilde{h}$.)

For a triple $\left(x_{0}, x_{1}, x_{2}\right)$ of vertices of $X$, the sign $\epsilon\left(x_{0}, x_{1}, x_{2}\right)$ is $1,-1$ or 0 depending on the orientation of the ideal triangle of $\mathbb{H}^{2}$ with vertices $\rho\left(p\left(x_{i}\right)\right) \bar{q}$ (just as in Subsection 4.2). Proposition 4.1 ensures that $\epsilon \in Z_{b}^{2}(\Gamma \curvearrowright X)^{\Gamma}$.

Let $t \in \Gamma$ be the stable letter of the HNN extension $\Gamma=\Gamma_{0} * \psi_{*}$. As in Section 4.3 we can define a simplicial cochain $F$ by setting

$$
F\left(\left[x_{0}, x_{1}, x_{2}\right]\right)=\frac{1}{3} \epsilon\left(x_{0}, x_{1}, x_{2}\right) \sum_{i=0}^{2} \theta\left(x_{i}\right) .
$$

In this context we have the following:

Lemma 5.6. The simplicial cochain $F$ satisfies:

(1) $\left|F\left(\partial\left[x_{0}, x_{1}, x_{2}, x_{3}\right]\right)\right| \leq 2 \kappa$ for any 3-simplex $\left[x_{0}, x_{1}, x_{2}, x_{3}\right] \in \mathcal{X}$;

(2) $\delta F$ is $\Gamma$-invariant.

Proof. The same computation as in Lemma 4.2 gives that, up to reordering the vertices $x_{i}$, either $F\left(\partial\left[x_{0}, x_{1}, x_{2}, x_{3}\right]\right)=0$ or $F\left(\partial\left[x_{0}, x_{1}, x_{2}, x_{3}\right]\right)=$ $\theta\left(x_{2}\right)-\theta\left(x_{3}\right)$, or $F\left(\partial\left[x_{0}, x_{1}, x_{2}, x_{3}\right]\right)=\left(\theta\left(x_{0}\right)-\theta\left(x_{1}\right)\right)+\left(\theta\left(x_{2}\right)-\theta\left(x_{3}\right)\right)$. Since $\theta$ is 1-Lipschitz and $d\left(x_{i}, x_{j}\right) \leq \kappa,(1)$ follows.

(2) follows from the description of $F\left(\partial\left[x_{0}, x_{1}, x_{2}, x_{3}\right]\right)$ we have just given, and from the fact that $\epsilon$ is $\Gamma$-invariant and $\theta(t \cdot x)=\theta(x)+\tau(\psi)$.

The $\Gamma_{0}$-invariant primitive of the volume form is the evaluation of $F$ on fillings of simplices:

$$
\alpha\left(x_{0}, x_{1}, x_{2}\right)=F\left(\varphi\left(x_{0}, x_{1}, x_{2}\right)\right) .
$$

An immediate consequence of Lemma 5.5 (2) and Lemma 5.6(1) is:

Lemma 5.7. The defect of $\alpha$ is uniformly bounded:

$$
\|\delta \alpha\|_{\infty} \leq 2 \kappa T_{3} .
$$

5.3. The volume estimate. In order to estimate the simplicial volume, recall that the Rips complex $\mathcal{X}$ is contractible, in particular we can choose a simplicial chain $S \in C_{2}^{\Delta}(\mathcal{X})_{\text {red, } \Gamma_{0}}$ representing the fundamental class of a fiber.

Lemma 5.8. If $S=\sum c(\sigma) \sigma$ then

$$
\sum_{\sigma} c(\sigma) \epsilon(\sigma)=-2 \chi(\Sigma) .
$$


Proof. The oriented area of a hyperbolic triangle is $\pi$ times the orientation cocycle $\epsilon$. Using Gauss-Bonnet this implies that the pairing of $\epsilon$ with a fundamental class $S$ of the surface $\Sigma$ is equal to $-2|\chi(\Sigma)|$, that is the volume of the surface divided by $\pi$.

Lemma 5.9. There exists a simplicial chain $M \in C_{3}\left(\Gamma_{0} \curvearrowright X\right)_{\text {red, } \Gamma_{0}}$ with $\partial M=t \cdot S-S$. The image of $M$ in $C_{3}(\Gamma \curvearrowright X)_{r e d, \Gamma}$ represents the fundamental class $\left[M_{\psi}\right] \in H_{3}\left(M_{\psi}, \mathbb{R}\right)$.

Proof. Denote by $\bar{M}_{\psi}$ the infinite cyclic cover of $M_{\psi}$. Since $\mathcal{X}$ is contractible, the orbit maps define maps $C_{*}\left(\Gamma_{0}\right)_{\mathrm{red}, \Gamma_{0}} \rightarrow C_{*}\left(\Gamma_{0} \curvearrowright X\right)_{\mathrm{red}, \Gamma_{0}}$ and $C_{*}\left(\Gamma_{0}\right)_{\text {red, } \Gamma_{0}} \rightarrow C_{*}\left(\bar{M}_{\psi}\right)_{\text {red }}$ inducing isomorphisms in homology. Each complex is endowed with a $\mathbb{Z}$-action (with the positive generators of $\mathbb{Z}$ acting as $\psi_{*}$ on $C_{*}\left(\Gamma_{0}\right)_{\text {red, } \Gamma_{0}}$, and as the positive generator of the deck transformation group of $\bar{M}_{\psi}$ on $\left.C_{*}\left(\bar{M}_{\psi}\right)_{\text {red }}\right)$ and the isomorphisms are equivariant with respect to these actions.

Using these isomorphisms the lemma follows from the corresponding statement for the topological counterpart, namely that if $[\Sigma] \in H_{2}\left(\bar{M}_{\psi}, \mathbb{R}\right)$ is represented by a fiber and $\bar{t}$ is a generator of the deck group, then $\bar{t}[\Sigma]-[\Sigma]$ is the boundary of a 3-cycle projecting to the fundamental class of $M_{\psi}$.

Recall that the simplicial volume $\|N\|$ of a closed oriented manifold $N$ is the $\ell^{1}$-seminorm of its real fundamental class. A fundamental result by Gromov and Thurston (see e.g. Thu79]) states that there exists a positive constant $v_{n}$ only depending on $n$ such that $\operatorname{vol}(N)=v_{n}\|N\|$ for every closed orientable hyperbolic $n$-manifold $N$. Therefore, Theorem 5.1 is an immediate consequence of the following:

Proposition 5.10.

$$
\left\|M_{\psi}\right\| \geq \frac{-2 \tau(\psi) \chi\left(\Sigma_{g}\right)}{\kappa T_{3}} .
$$

Proof. Since $M$ represents $\left[M_{\psi}\right]$, we have

$$
|\delta \alpha(M)| \leq\left\|\left[M_{\psi}\right]\right\|_{1} \cdot\|[\delta \alpha]\|_{\infty}=\left\|M_{\psi}\right\| \cdot\|[\delta \alpha]\|_{\infty} \leq 2 \kappa T_{3}\left\|M_{\psi}\right\| .
$$

By construction, for any $x \in X$ we have $\theta(t \cdot x)=\tau(\psi)+\theta(x)$. In particular, for any 3-simplex $\sigma=\left[x_{0}, x_{1}, x_{2}\right] \in \mathcal{X}$ we get $\alpha(t \cdot \sigma)-\alpha(\sigma)=$ $\epsilon(\sigma) \tau(\psi)$. This implies

$$
\delta \alpha(M)=\alpha(\partial M)=\tau(\psi) \sum_{\sigma} c(\sigma) \epsilon(\sigma)=-2 \chi\left(\Sigma_{g}\right) \tau(\psi) .
$$

The conclusion now follows from (4) and (5).

\section{REFERENCES}

[BB16] Jeffrey F. Brock and Kenneth W. Bromberg. Inflexibility, Weil-Peterson distance, and volumes of fibered 3-manifolds. Math. Res. Lett., 23(3):649-674, 2016.

[BBF16] M. Bestvina, K. Bromberg, and K. Fujiwara. Bounded cohomology with coefficients in uniformly convex Banach spaces. Comment. Math. Helv., 91:203-218, 2016. 
[Ber78] Lipman Bers. An extremal problem for quasiconformal mappings and a theorem by Thurston. Acta Math., 141(1-2):73-98, 1978.

[BF02] M. Bestvina and K. Fujiwara. Bounded cohomology of subgroups of mapping class groups. Geom. Topol., 6:69-89 (electronic), 2002.

[BH99a] M. R. Bridson and A. Haefliger. Metric spaces of non-positive curvature, volume 319 of Grundlehren der Mathematischen Wissenschaften. Springer-Verlag, Berlin, 1999.

[BH99b] Martin R. Bridson and André Haefliger. Metric spaces of non-positive curvature, volume 319 of Grundlehren der Mathematischen Wissenschaften [Fundamental Principles of Mathematical Sciences]. Springer-Verlag, Berlin, 1999.

[BI07] M. Burger and A. Iozzi. Bounded differential forms, generalized Milnor-Wood inequality and an application to deformation rigidity. Geom. Dedicata, 125:1-23, 2007.

[Bow12] B. H. Bowditch. Relatively hyperbolic groups. Internat. J. Algebra Comput., 22(3):1250016, 66, 2012.

[Bro81] R. Brooks. Some remarks on bounded cohomology. In Princeton Univ. Press, editor, Riemann surfaces and related topics: Proceedings of the 1978 Stony Brook Conference (State Univ. New York, Stony Brook, N.Y., 1978), volume 97 of Ann. of Math. Stud., Princeton, N.J., 1981.

[Bro03a] Jeffrey F. Brock. The Weil-Petersson metric and volumes of 3-dimensional hyperbolic convex cores. J. Amer. Math. Soc., 16(3):495-535, 2003.

[Bro03b] Jeffrey F. Brock. Weil-Petersson translation distance and volumes of mapping tori. Comm. Anal. Geom., 11(5):987-999, 2003.

[CFI16] Indira Chatterji, Talia Fernós, and Alessandra Iozzi. The median class and superrigidity of actions on CAT(0) cube complexes. J. Topol., 9(2):349-400, 2016. With an appendix by Pierre-Emmanuel Caprace.

[DGO16] F. Dahmani, V. Guirardel, and D. Osin. Hyperbolically embedded subgroups and rotating families in groups acting on hyperbolic spaces. Mem. Amer. Math. Soc., 245(1156), 2016.

[EF97] D. Epstein and K. Fujiwara. The second bounded cohomology of word-hyperbolic groups. Topology, 36:1275-1289, 1997.

[Far98] B. Farb. Relatively hyperbolic groups. Geom. Funct. Anal., 8:810-840, 1998.

[FM02] B. Farb and L. Mosher. The geometry of surface-by-free groups. Geom. Funct. Anal., 12(5):915-963, 2002.

[FM11] K. Fujiwara and J. F. Manning. Simplicial volume and fillings of hyperbolic manifolds. Algebr. Geom. Topol., 11:2237-2264, 2011.

[FPS15] R. Frigerio, M. B. Pozzetti, and A. Sisto. Extending higher-dimensional quasicocycles. J. Topol., 8:1123-1155, 2015.

[Fra] F. Franceschini. A characterization of relatively hyperbolic groups via bounded cohomology. arXiv:1505.04465.

[Fuj98] K. Fujiwara. The second bounded cohomology of a group acting on a Gromovhyperbolic space. Proc. London Math. Soc., 76:70-94, 1998.

[Fuj00] K. Fujiwara. The second bounded cohomology of an amalgamated free product of groups. Trans. Amer. Math. Soc., 352:1113-1129, 2000.

[GM08] D. Groves and J. F. Manning. Dehn filling in relatively hyperbolic groups. Israel J. Math., 168:317-429, 2008.

[Gro82] M. Gromov. Volume and bounded cohomology. Inst. Hautes Études Sci. Publ. Math., 56:5-99, 1982.

[Gro99] M. Gromov. Metric structures for Riemannian and non-Riemannian spaces, volume 152 of Progress in Mathematics. Birkhäuser, 1999. with appendices by M. Katz, P. Pansu, and S. Semmes, translated by S.M. Bates.

[GS14] Dominik Gruber and Alessandro Sisto. Infinitely presented graphical small cancellation groups are acylindrically hyperbolic. 2014. 
[Ham08] U. Hamenstädt. Bounded cohomology and isometry groups of hyperbolic spaces. J. Eur. Math. Soc., 10:315-349, 2008.

[HO13] M. Hull and D. Osin. Induced quasicocycles on groups with hyperbolically embedded subgroups. Algebr. Geom. Topol., 13:2635-2665, 2013.

[Iva87] N. V. Ivanov. Foundation of the theory of bounded cohomology. J. Soviet. Math., 37:1090-1114, 1987.

[Iva90] N. V. Ivanov. The second bounded cohomology group. J. Soviet Math., 52:28222824, 1990.

[KK15] S. Kim and I. Kim. Bounded cohomology and the cheeger isoperimetric constant. Geom. Dedicata, 179:1-20, 2015.

[KL07] Richard P. Kent, IV and Christopher J. Leininger. Subgroups of mapping class groups from the geometrical viewpoint. In In the tradition of Ahlfors-Bers. IV, volume 432 of Contemp. Math., pages 119-141. Amer. Math. Soc., Providence, RI, 2007.

[KM14] S. Kojima and G. McShane. Normalized Entropy versus Volume. ArXiv e-prints, November 2014.

[Min01] I. Mineyev. Straightening and bounded cohomology of hyperbolic groups. Geom. Funct. Anal., 11:807-839, 2001.

[MM85] S. Matsumoto and S. Morita. Bounded cohomology of certain groups of homeomorphisms. Proc. Amer. Math. Soc., 94:539-544, 1985.

[MM00] H. A. Masur and Y. N. Minsky. Geometry of the complex of curves. II. Hierarchical structure. Geom. Funct. Anal., 10(4):902-974, 2000.

[MO13] A. Minasyan and D. Osin. Acylindrical hyperbolicity of groups acting on trees. ArXiv 1310.6289, October 2013.

[Mon01] N. Monod. Continuous bounded cohomology of locally compact groups. Number 1758 in Lecture notes in Mathematics. Springer-Verlag, Berlin, 2001.

[MR08] Mahan Mj and Lawrence Reeves. A combination theorem for strong relative hyperbolicity. Geom. Topol., 12(3):1777-1798, 2008.

[MS06] N. Monod and Y. Shalom. Orbit equivalence rigidity and bounded cohomology. Ann. of Math. (2), 164:825-878, 2006.

[MS17] J. Maher and A. Sisto. Random subgroups of acylindrically hyperbolic groups and hyperbolic embeddings. 2017.

[NM04] Y. Shalom N. Monod. Cocycle superrigidity and bounded cohomology for negatively curved spaces. J. Differential Geom., 67:395-455, 2004.

[Osi13] D. Osin. Acylindrically hyperbolic groups. ArXiv 1304.1246, April 2013.

[Osi15] D. Osin. On acylindrical hyperbolicity of groups with positive first $\ell^{2}$-Betti number. Bull. Lond. Math. Soc., 47:725-730, 2015.

[Ota96] J. P. Otal. Le théorème d'hyperbolisation pour les variétés fibrées de dimension 3. Astérisque, 235:x+159 pp., 1996.

[Raf05] Kasra Rafi. A characterization of short curves of a Teichmüller geodesic. Geom. Topol., 9:179-202, 2005.

[Raf07] Kasra Rafi. A combinatorial model for the Teichmüller metric. Geom. Funct. Anal., 17(3):936-959, 2007.

[Sis11] A. Sisto. Contracting elements and random walks. ArXiv 1112.2666. December 2011.

[Som97a] T. Soma. Bounded cohomology and topologically tame Kleinian groups. Duke Math. J., 88:357-370, 1997.

[Som97b] T. Soma. Bounded cohomology of closed surfaces. Topology, 36:1221-1246, 1997.

[Som97c] T. Soma. The zero-norm subspace of bounded cohomology. Comment. Math. Helv., 72:582-592, 1997.

[Som98] T. Soma. Existence of non-Banach bounded cohomology. Topology, 37:179-193, 1998. 
THE ZERO NORM SUBSPACE FOR ACYLINDRICALLY HYPERBOLIC GROUPS 46

[Thu79] W. P. Thurston. The geometry and topology of 3-manifolds. Princeton, 1979. mimeographed notes.

[Wie12] A. Wienhard. Remarks on and around bounded differential forms. Pure Appl. Math. Q., 8:479-496, 2012.

Karlsruher Institut für Technologie (KIT), Fakultät für Mathematik, InStitut für Algebra und Geometrie Englerstrasse. 2, 76131 Karlsruhe, DeutschLAND

E-mail address: federico.franceschini@kit.edu

Dipartimento di Matematica, Università di Pisa, Largo B. Pontecorvo 5, 56127 PisA, ITALY

E-mail address: frigerio@dm.unipi.it

Mathematics Institute, Zeeman Bullding, University of Warwick, Coventry CV4 7AL United Kingdom

E-mail address: B.Pozzetti@Warwick.ac.uk

Department Mathematik, ETH Zürich, RÄmistrasse 101, CH-8092 Zürich, SWITZERLAND

E-mail address: sisto@math.ethz.ch 